Prepared for the U.S. Department of Energy under Contract DE-AC05-76RL01830

\title{
Statistics for the Relative Detectability of Chemicals in Weak Gaseous Plumes in LWIR Hyperspectral Imagery
}

CN Metoyer

SJ Walsh

MF Tardiff

LK Chilton

October 2008

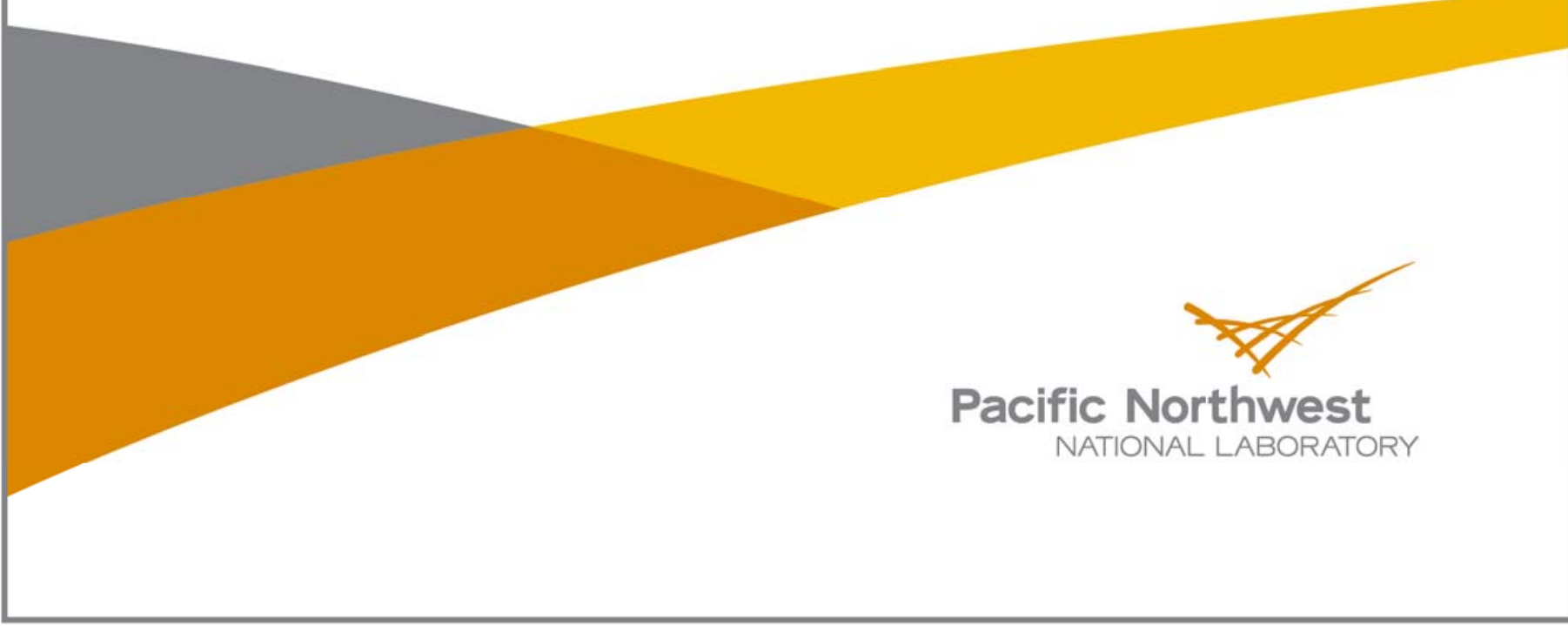




\title{
DISCLAIMER
}

This report was prepared as an account of work sponsored by an agency of the United States Government. Neither the United States Government nor any agency thereof, nor Battelle Memorial Institute, nor any of their employees, makes any warranty, express or implied, or assumes any legal liability or responsibility for the accuracy, completeness, or usefulness of any information, apparatus, product, or process disclosed, or represents that its use would not infringe privately owned rights. Reference herein to any specific commercial product, process, or service by trade name, trademark, manufacturer, or otherwise does not necessarily constitute or imply its endorsement, recommendation, or favoring by the United States Government or any agency thereof, or Battelle Memorial Institute. The views and opinions of authors expressed herein do not necessarily state or reflect those of the United States Government or any agency thereof.

\author{
PACIFIC NORTHWEST NATIONAL LABORATORY \\ operated by \\ BATTELLE \\ for the \\ UNITED STATES DEPARTMENT OF ENERGY \\ under Contract DE-AC05-76RL01830
}

Printed in the United States of America
Available to DOE and DOE contractors from the Office of Scientific and Technical Information,
P.O. Box 62, Oak Ridge, TN 37831-0062;
ph: (865) 576-8401
fax: $(865)$ 576-5728
email: reports@adonis.osti.gov

\begin{abstract}
Available to the public from the National Technical Information Service, U.S. Department of Commerce, 5285 Port Royal Rd., Springfield, VA 22161 ph: (800) 553-6847 fax: $(703) 605-6900$ email: orders@ntis.fedworld.gov online ordering: http://www.ntis.gov/ordering.htm
\end{abstract}

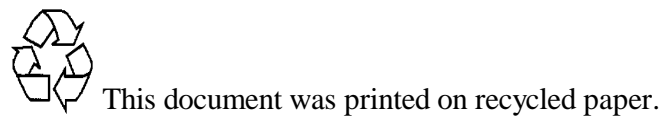




\title{
Statistics for the Relative Detectability of Chemicals in Weak Gaseous Plumes in LWIR Hyperspectral Imagery
}

\author{
CN Metoyer \\ SJ Walsh \\ MF Tardiff \\ LK Chilton
}

October 2008

Prepared for

the U.S. Department of Energy

Under Contract DE-AC05-76RL01830

Pacific Northwest National Laboratory

Richland, Washington 99352 


\section{Executive Summary}

The detection and identification of weak gaseous plumes using thermal imaging data is complicated by many factors. These include variability due to atmosphere, ground and plume temperature, and background clutter. This report presents an analysis of one formulation of the physics-based model that describes the at-sensor observed radiance. The motivating question for the analyses performed in this report is as follows. Given a set of backgrounds, is there a way to predict the background over which the probability of detecting a given chemical will be the highest? Two statistics were developed to address this question. These statistics incorporate data from the long-wave infrared band to predict the background over which chemical detectability will be the highest. These statistics can be computed prior to data collection. As a preliminary exploration into the predictive ability of these statistics, analyses were performed on synthetic hyperspectral images. Each image contained one chemical (either carbon tetrachloride or ammonia) spread across six distinct background types. The statistics were used to generate predictions for the background ranks. Then, the predicted ranks were compared to the empirical ranks obtained from the analyses of the synthetic images. For the simplified images under consideration, the predicted and empirical ranks showed a promising amount of agreement. One statistic accurately predicted the best and worst background for detection in all of the images. Future work may include explorations of more complicated plume ingredients, background types, and noise structures. 


\section{Contents}

1 Introduction ........................ 1

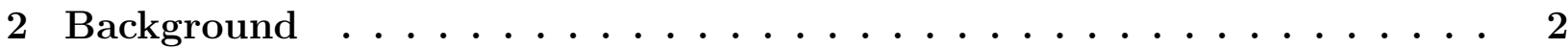

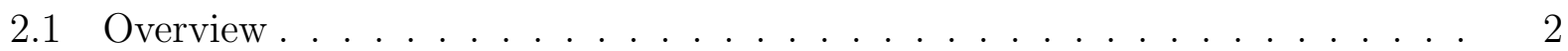

2.2 Radiance Model . . . . . . . . . . . . . . . . . . . . . . . 2

2.3 An Application to Hyperspectral Imagery . . . . . . . . . . . . . . . . . . . 4

3 A Hypothesis-Test-Based Definition of Detection . . . . . . . . 5

3.1 Overview . . . . . . . . . . . . . . . . . . . . . 5

3.2 Relevant Point Estimates . . . . . . . . . . . . . . . . . . . . . . . 5

3.3 A Hypothesis Test for Chemical Detection . . . . . . . . . . . . . 6

3.4 The Power of the Test . . . . . . . . . . . . . . . . . . . . . . 8

3.5 Statistics for Background Ranking . . . . . . . . . . . . . . . . . . . . . 11

4 Experimental Methods ..................... 12

4.1 Overview . . . . . . . . . . . . . . . . . . . . . . 12

4.2 Generation of Synthetic Hyperspectral Images . . . . . . . . . . . . . . . . . 13

4.3 Analyses Performed on Synthetic Images . . . . . . . . . . . . . . . . . . . . 14

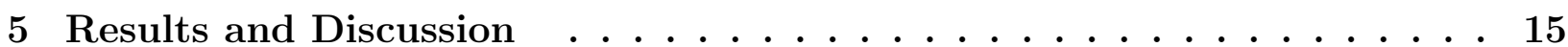

5.1 Results for the $\mathrm{CCl}_{4}$ Images . . . . . . . . . . . . . . . . . . . . . . 15

5.2 Results for the $\mathrm{NH}_{3}$ Images $\ldots \ldots \ldots \ldots$. . . . . . . . . . . . . 17

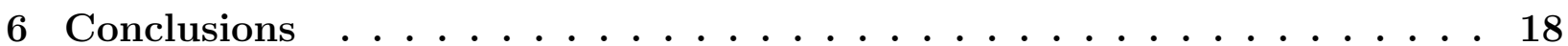

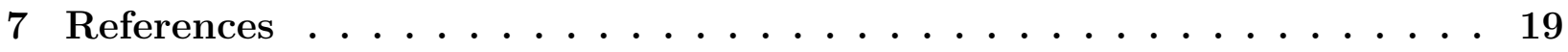




\section{List of Figures}

1 (a) Absorbance spectrum for $\mathrm{CCl}_{4}$, (b) Absorbance spectrum for $\mathrm{NH}_{3}$, Representative emissivity spectra for six selected materials, and (d) 1976 US Standard atmospheric transmissivity spectrum. . . . . . . . . . . .

2 Example of a broadband image for $\mathrm{CCl}_{4}$ using synthetic data from IR-SAGE. This image has been enhanced to better show the gradations between background materials and concentration path-lengths. . . . . . . . . . . .

3 Graphical results for the IR-SAGE images containing $\mathrm{CCl}_{4}$ with $T_{p}=310 \mathrm{~K}$ and $T_{g}=300 K$ : (a) $M_{1}$ versus background material, (b) $M_{2}$ versus background material, (c) predicted power function, and (d) empirical detection curves. Note that plots (a) and (c) incorporate covariance matrices computed from the $0 \mathrm{ppm}-m$ pixels. . . . . . . . . . . . . . .

4 Graphical results for the IR-SAGE images containing $\mathrm{CCl}_{4}$ with $T_{p}=T_{g}=$ $300 K$ : (a) $M_{1}$ versus background material, (b) $M_{2}$ versus background material, (c) predicted power function, and (d) empirical detection curves. Note that plots (a) and (c) incorporate covariance matrices computed from the 0

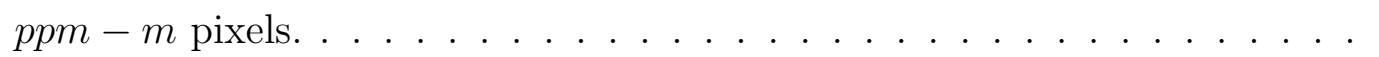

5 Graphical results for the IR-SAGE images containing $\mathrm{CCl}_{4}$ with $T_{p}=290 \mathrm{~K}$ and $T_{g}=300 K$ : (a) $M_{1}$ versus background material, (b) $M_{2}$ versus background material, (c) predicted power function, and (d) empirical detection curves. Note that plots (a) and (c) incorporate covariance matrices computed from the $0 \mathrm{ppm}-m$ pixels. . . . . . . . . . . . . . .

6 Graphical results for the IR-SAGE images containing $\mathrm{NH}_{3}$ with $T_{p}=310 \mathrm{~K}$ and $T_{g}=300 \mathrm{~K}$ : (a) $M_{1}$ versus background material, (b) $M_{2}$ versus background material, (c) predicted power function, and (d) empirical detection curves. Note that plots (a) and (c) incorporate covariance matrices computed from the $0 \mathrm{ppm}-m$ pixels. . . . . . . . . . . . . . . .

7 Graphical results for the IR-SAGE images containing $\mathrm{NH}_{3}$ with $T_{p}=T_{g}=$ $300 K$ : (a) $M_{1}$ versus background material, (b) $M_{2}$ versus background material, (c) predicted power function, and (d) empirical detection curves. Note that plots (a) and (c) incorporate covariance matrices computed from the 0 ppm $-m$ pixels. . . . . . . . . . . . . . . . . . . 
8 Graphical results for the IR-SAGE images containing $\mathrm{NH}_{3}$ with $T_{p}=290 \mathrm{~K}$ and $T_{g}=300 K$ : (a) $M_{1}$ versus background material, (b) $M_{2}$ versus background material, (c) predicted power function, and (d) empirical detection curves. Note that plots (a) and (c) incorporate covariance matrices computed from the $0 \mathrm{ppm}-m$ pixels. . . . . . . . . . . . . . 


\section{List of Tables}

1 Background ranking ( $1=$ best, $6=$ worst) implied by prediction metric $M_{1}$, prediction metric $M_{2}$, and the empirical detection proportions (for selected values of $k$ ) for $\mathrm{CCl}_{4}$ and $\mathrm{NH}_{3}$ where $T_{p}=310 K$ and $T_{g}=300 K \ldots \ldots$. .

2 Background ranking ( $1=$ best, $6=$ worst) implied by prediction metric $M_{1}$, prediction metric $M_{2}$, and the empirical detection proportions (for selected values of $k$ ) for $\mathrm{CCl}_{4}$ and $\mathrm{NH}_{3}$ where $T_{p}=T_{g}=300 K \ldots \ldots \ldots$.

3 Background ranking ( 1 =best, $6=$ worst) implied by prediction statistic $M_{1}$, prediction statistic $M_{2}$, and the empirical detection proportions (for selected values of $k$ ) for $\mathrm{CCl}_{4}$ and $\mathrm{NH}_{3}$ where $T_{p}=290 \mathrm{~K}$ and $T_{g}=300 \mathrm{~K} . \ldots$. . 


\section{Introduction}

For any estimation task, the ability to characterize unknowns depends on the delicate interplay between the underlying signal and the background noise. In the field of hyperspectral imagery, the characterization of gaseous plumes often includes the following three main tasks: detection, identification, and quantification [3]. Techniques for the characterization of gaseous plumes have been studied extensively $[2,3,4,8]$.

Many of these studies have focused on the characterization of the background variability where the term variability can include terms related to noise and clutter. The idea is that as the understanding of the background behavior improves, the ability to detect (identify, and quantify) gaseous plumes will also improve. As such, many of the current estimation procedures provide information about chemical detectability after data have already been collected. However, it would be helpful to have some information about detectability before data are collected. That is, if one were planning a mission to certain sites with the goal of detecting chemicals, it would be useful to have a mechanism in place that that could predict chemical detectability at the sites of interest.

Such a mechanism may be referred to as a mission planning tool (MPT). Site-specific information (such as atmospheric transmissivity, background temperature, background emissivity, and background clutter), chemical- and plume-specific information (such as chemical absorbance and plume temperature), and sensor-specific information (such as calibration corrections and response functions) can be used as inputs for the MPT. The MPT may output a variety of information. For example, if information from several sites is available, the MPT may be used to identify the site over which the relative probability of chemical detection is the highest. Further extensions of the MPT could include the actual calculation of the relative probability of detection at each site.

In this report, a preliminary framework for a MPT is proposed. The context for the MPT in this report is as follows. Suppose there is one chemical of interest and several background conditions over which the chemical is to be detected. The challenge is to be able to rankorder the background conditions from best to worst in terms of the ease with which the chemical of interest can be detected. Focusing on the characterization of weak gaseous plumes, two statistics (one main statistic and then a simplified version of the main statistic) are identified. These statistics serve as MPTs; i.e., tools that can be used to predict some 
aspect of detectability.

The statistics could also be used to rank-order a set of chemicals from easiest-to-detect to hardest-to-detect; however, this type of application is not discussed in this report. Also, plume detection is not addressed. Rather, the focus is on the detection of the signature of a given chemical in a plume. For the scenarios presented in this report, preliminary results from simplified synthetic hyperspectral images show that each of the identified statistics has the ability to predict the empirical (i.e. observed) rank-orders of the background conditions.

This report is organized as follows. Section 2 includes a review of the governing plume radiance model. Well-known assumptions are applied and a linear model for radiance is developed. In Section 3, a distinction is made between chemical detection and chemical identification. A hypothesis test for chemical detection is constructed. Computation of the power function for this test reveals statistics that appear to drive chemical detectability. Section 4 contains a description of experiments that were conducted in order to evaluate the performance of the predictive statistics. These experiments were conducted on simplified synthetic hyperspectral images. The results of the experiments are presented in Section 5 and a summary is presented in Section 6.

\section{Background}

\subsection{Overview}

Presented in this section is a review of the physics-based model that is used to characterize the radiance of a gaseous plume. At this preliminary stage, it is assumed that the gaseous plume contains exactly one chemical. Future work may include generalizations of the model for more than one chemical. Well-known assumptions are applied to the radiance model and a linear model for gas-plume radiance is developed.

\subsection{Radiance Model}

In order to gain insight into how background conditions affect the at-sensor observed plume signal, the three-layer physics-based radiance model is explored $[2,4,6]$. This model can 
be written as

$$
L_{o b s}(\nu)=\tau_{a}(\nu)\left(\left(1-\tau_{p}(\nu)\right) B\left(T_{p} ; \nu\right)+\tau_{p}(\nu) L_{g}(\nu)\right)+L_{u}(\nu)+e(\nu),
$$

where $L_{o b s}(\nu)$ represents observed radiance in $\mathrm{W} / \mathrm{cm}^{2} \times \mathrm{Sr} \times \mathrm{cm}^{-1}$ at wavenumber $\nu\left(\mathrm{cm}^{-1}\right)$, $\tau_{a}(\nu)$ and $\tau_{p}(\nu)$ are dimensionless quantities representing atmospheric and plume transmissivity, respectively, and are between 0 and $1, B(T ; \nu)$ has radiance units and is Planck's Blackbody function at wavenumber $\nu$ and temperature $T(K), T_{p}$ and $T_{g}$ are the temperatures of the plume and gas, respectively, and $L_{g}(\nu)$ and $L_{u}(\nu)$ represent ground-leaving radiance and atmospheric-upwelling radiance, respectively. Following the convention of Burr $[1,2], e(\nu)$ represents any unmodeled effects and instrument noise.

Following the convention of Burr [2] and Schott [8], the ground-leaving radiance is modeled as

$$
L_{g}(\nu)=\epsilon_{g}(\nu) B\left(T_{g} ; \nu\right)
$$

where $\epsilon_{g}(\nu)$ is a dimensionless quantity (between 0 and 1) representing the emissivity of the ground at wavenumber $\nu$. It is important to note that this formulation ignores the reflected atmospheric downwelling radiance. This assumption is reasonable because the contribution of the reflected radiance to the observed signal is negligible in the LWIR band [8].

The Beer-Bourger-Lambert Law [5] gives an explicit expression for the transmissivity of a gas in terms of the chemical effluent's concentration path-length, $c$, where $c$ is measured in parts-per-million-meter, denoted $p p m-m$. The expression is

$$
\tau_{p}(\nu)=\exp \{-A(\nu) c\}
$$

where $A(\nu)$ is the absorbance coefficient of the gas at wavenumber $\nu$ and has units of inverse ppm-m. For optically thin plumes [2], this term is well-approximated by a Taylor series expansion to the linear term, i.e.,

$$
\tau_{p}(\nu) \approx 1-A(\nu) c
$$

Substitution of Equations (2) and (4) into Equation (1) yields the following working gas plume linear model (GPLM):

$$
L_{o b s}(\nu) \approx \tau_{a}(\nu)\left(B\left(T_{p} ; \nu\right)-\epsilon_{g}(\nu) B\left(T_{g} ; \nu\right)\right) A(\nu) c+\tau_{a}(\nu) \epsilon_{g}(\nu) B\left(T_{g} ; \nu\right)+L_{u}(\nu)+e(\nu) .
$$


By collecting terms across the spectral dimension, a vector-form of the GPLM can be formed. Let the wavenumbers under consideration be denoted $\nu_{1}, \nu_{2}, \ldots, \nu_{n}$, and let $\boldsymbol{\nu}=\left(\nu_{1}, \ldots, \nu_{n}\right)$ be the collection of these wavenumbers. Effectively, this means that there are $n$ spectral channels. Then, Equation (5) can be expressed as

$$
\mathbf{L}_{o b s} \approx \boldsymbol{\tau}_{a} \odot\left(\mathbf{B}\left(T_{p}\right)-\boldsymbol{\epsilon}_{g} \odot \mathbf{B}\left(T_{g}\right)\right) \odot \mathbf{A} c+\boldsymbol{\tau}_{a} \odot \boldsymbol{\epsilon}_{g} \odot \mathbf{B}\left(T_{g}\right)+\mathbf{L}_{u}+\mathbf{e}
$$

where all bold terms are $n \times 1$ vectors and $\odot$ denotes the Hadamard product (element-wise multiplication). Examination of Equation (6) shows that the radiance due to the chemical plume is:

$$
\boldsymbol{\tau}_{a} \odot\left(\mathbf{B}\left(T_{p}\right)-\boldsymbol{\epsilon}_{g} \odot \mathbf{B}\left(T_{g}\right)\right) \odot \mathbf{A} c .
$$

In previous work, the term $\left(\mathbf{B}\left(T_{p}\right)-\boldsymbol{\epsilon}_{g} \odot \mathbf{B}\left(T_{g}\right)\right)$ has been referred to as the TemperatureEmissivity contrast [10].

\subsection{An Application to Hyperspectral Imagery}

Hyperspectral images may contain on-plume pixels (pixels that have a gas plume that influences the signal) and off-plume pixels (pixels that do not have a gas plume influencing the signal) [2]. Using Equation (6) as a guide, the at-sensor observed radiance for an on-plume pixel, indexed $i$, can be modeled as:

$$
\mathbf{L}_{o n}^{i}=\boldsymbol{\tau}_{a}^{i} \odot\left(\mathbf{B}\left(T_{p}^{i}\right)-\boldsymbol{\epsilon}_{g}^{i} \odot \mathbf{B}\left(T_{g}^{i}\right)\right) \odot \mathbf{A} c^{i}+\boldsymbol{\tau}_{a}^{i} \odot \boldsymbol{\epsilon}_{g}^{i} \odot \mathbf{B}\left(T_{g}^{i}\right)+\mathbf{L}_{u}^{i}+\mathbf{e}^{i}
$$

and the at-sensor observed radiance for an off-plume pixel, indexed $j$, can be modeled as:

$$
\mathbf{L}_{o f f}^{j}=\boldsymbol{\tau}_{a}^{j} \odot \boldsymbol{\epsilon}_{g}^{j} \odot \mathbf{B}\left(T_{g}^{j}\right)+\mathbf{L}_{u}^{j}+\mathbf{e}^{j} .
$$

Off-plume pixels are also sometimes called background pixels and the radiance in off-plume pixels is often referred to as background radiance. In traditional estimation procedures, the background radiance is subtracted from the radiance found in the on-plume pixels $[1,2,3]$. This is done to isolate the radiance that is solely due to the chemical plume. At this preliminary stage, it is assumed that atmospheric transmissivity and up-welling radiance are constant across all pixels. Then, taking the average of $\mathbf{L}_{\text {off }}^{j}$ across all background pixels 
yields

$$
\overline{\mathbf{L}_{o f f}}=\boldsymbol{\tau}_{a} \odot \overline{\boldsymbol{\epsilon}_{g} \odot \mathbf{B}\left(T_{g}\right)}+\mathbf{L}_{u}+\overline{\mathbf{e}},
$$

and $\overline{\mathbf{L}_{o f f}}$ is viewed as the average background radiance. The background radiance in Equation (10) is subtracted from the on-plume radiance in Equation (8) yielding

$$
\mathbf{L}_{o n}^{i}-\overline{\mathbf{L}_{o f f}}=\boldsymbol{\tau}_{a} \odot\left(\mathbf{B}\left(T_{p}^{i}\right)-\boldsymbol{\epsilon}_{g}^{i} \odot \mathbf{B}\left(T_{g}^{i}\right)\right) \odot \mathbf{A} c^{i}+\boldsymbol{\eta}^{i}
$$

where

$$
\boldsymbol{\eta}^{i}=\boldsymbol{\tau}_{a} \odot\left(\boldsymbol{\epsilon}_{g}^{i} \odot \mathbf{B}\left(T_{g}^{i}\right)-\overline{\boldsymbol{\epsilon}_{g} \odot \mathbf{B}\left(T_{g}\right)}\right)+\left(\mathbf{e}^{i}-\overline{\mathbf{e}}\right)
$$

contains clutter and noise terms. Then, dropping the pixel-identifying index $i$, the radiance in each pixel is modeled according to the following mean-corrected GPLM

$$
\mathbf{L}_{o n}-\overline{\mathbf{L}_{o f f}}=\boldsymbol{\tau}_{a} \odot\left(\mathbf{B}\left(T_{p}\right)-\boldsymbol{\epsilon}_{g} \odot \mathbf{B}\left(T_{g}\right)\right) \odot \mathbf{A} c+\boldsymbol{\eta}
$$

and it is assumed that $E(\boldsymbol{\eta})=\mathbf{0}$ and $\operatorname{Var}(\boldsymbol{\eta})=\Sigma_{g}$ where $\Sigma_{g}$ is an $n \times n$ background covariance matrix.

\section{A Hypothesis-Test-Based Definition of Detection}

\subsection{Overview}

In this section, the gas plume linear model developed in Section 2 is used to construct a hypothesis-test-based definition of detection. By computing the power of the hypothesis test, a statistic that drives chemical detectability is isolated. When given a set of background conditions, it is shown that this statistic can be used to rank-order the background conditions on the basis of chemical detectability.

\subsection{Relevant Point Estimates}

Let the absorption spectrum for chemical $j$ be denoted $\mathbf{A}_{j}$. Then, Equation (13) can be generically written as

$$
\mathbf{r}=\mathbf{x}_{j} \beta_{j}+\boldsymbol{\eta},
$$


where $\mathbf{r}=\mathbf{L}_{o n}-\overline{\mathbf{L}_{o f f}}$ is an $n \times 1$ vector of mean-centered at-sensor observed radiances. Following the convention of Burr [2], the $n \times 1$ vector $\mathbf{x}_{j}$ given by

$$
\mathbf{x}_{j}=\boldsymbol{\tau}_{a} \odot\left(\mathbf{B}\left(T_{p}\right)-\boldsymbol{\epsilon}_{g} \odot \mathbf{B}\left(T_{g}\right)\right) \odot \mathbf{A}_{j}
$$

is called the chemical signature and $\beta_{j}$ is viewed as the amount of chemical signature. For the work presented in this report, the sign or nature of $\beta_{j}$ is not investigated; however, these topics may be explored in future work.

Given $\mathbf{x}_{j}$ (that is, given $\boldsymbol{\tau}_{a}, T_{p}, T_{g}, \boldsymbol{\epsilon}_{g}$, and $\mathbf{A}_{j}$ ), the generalized least-squares estimator (GLSE) for $\beta_{j}$ is

$$
\widetilde{\beta}_{j}=\left(\mathbf{x}_{j}^{\prime} \Sigma_{g}^{-1} \mathbf{x}_{j}\right)^{-1} \mathbf{x}_{j}^{\prime} \Sigma_{g}^{-1} \mathbf{r}
$$

and this estimator has variance $\gamma_{j}$ where

$$
\gamma_{j} \equiv \operatorname{Var}\left(\widetilde{\beta}_{j}\right)=\left(\mathbf{x}_{j}^{\prime} \Sigma_{g}^{-1} \mathbf{x}_{j}\right)^{-1}
$$

In Equations (16) and (17), $\Sigma_{g}$ is unknown and must be estimated. In traditional analyses, an estimate for $\Sigma_{g}$ is computed from the off-plume pixels in the hyperspectral image that is being analyzed. However, in terms of the mission planning perspective, there is no "current" image from which an estimate can be computed. Fortunately, a potential solution exists. If there were previous fly-bys at the location of interest, then a covariance library may be available and an estimate for $\Sigma_{g}$ may be gleaned from this library. Alternatively, one may obtain an estimate of $\Sigma_{g}$ from domain experts. When absolutely no information is available, one may consider using an identity matrix as a temporary estimate for $\Sigma_{g}$. Thus, provided that a covariance matrix estimate, $\widehat{\Sigma}_{g}$, is available, the updated estimates are

$$
\widehat{\beta}_{j}=\left(\mathbf{x}_{j}^{\prime} \widehat{\Sigma}_{g}^{-1} \mathbf{x}_{j}\right)^{-1} \mathbf{x}_{j}^{\prime} \widehat{\Sigma}_{g}^{-1} \mathbf{r}
$$

and

$$
\left.\widehat{\gamma}_{j} \equiv \widehat{\operatorname{Var}\left(\widehat{\beta}_{j}\right.}\right)=\left(\mathbf{x}_{j}^{\prime} \widehat{\Sigma}_{g}^{-1} \mathbf{x}_{j}\right)^{-1}
$$

\subsection{A Hypothesis Test for Chemical Detection}

Using Equation (18), a point estimate for the amount of signature associated with chemical $j$ can be obtained; to evaluate the significance of this result (i.e. to see if the value appears 
to be different from zero), a $t$-test can be used [7]. Of interest are the hypotheses

$$
\begin{aligned}
& H_{o} \text { : The signature for chemical } j \text { is not present. } \\
& H_{a} \text { : The signature for chemical } j \text { is present. }
\end{aligned}
$$

where $H_{o}$ is called the null hypothesis and $H_{a}$ is called the alternative hypothesis. A test of these hypotheses is equivalent to testing the following set of hypotheses:

$$
\begin{array}{ll}
H_{o} & : \quad \beta_{j}=0 \\
H_{a} & : \quad \beta_{j} \neq 0 .
\end{array}
$$

Here, a two-tailed test is used. Future work may include studies of other types of tests; however, those topics are not covered in this work.

In the absence of other information, it is assumed that the errors follow a normal distribution, i.e., $\boldsymbol{\eta} \sim N\left(\mathbf{0}, \Sigma_{g}\right)$. Then, the appropriate test statistic is $t^{*}$ given by

$$
t^{*}=\frac{\widehat{\beta}_{j}}{\sqrt{\widehat{\operatorname{Var}\left(\widehat{\beta}_{j}\right)}}}=\frac{\widehat{\beta}_{j}}{\sqrt{\widehat{\gamma}_{j}}},
$$

where Equation (19) has been applied [7]. Small values of $t^{*}$ provide evidence in support of the null hypothesis while large values of $t^{*}$ provide evidence in support of the alternative hypothesis.

The model in Equation (14) is a type of no-intercept simple linear regression model. Therefore, the degree of freedom, $d f$, for the $t$-test is $n-1$ since only one parameter (the slope term) is being estimated [7]. Under the null hypothesis, $t^{*}$ follows a $t$-distribution with $d f=n-1$. Let $t_{1-\alpha / 2 ; n-1}$ be the value such that the probabilty that a $t$-distributed random variable with $d f=n-1$ is smaller than $t_{1-\alpha / 2 ; n-1}$ is $1-\alpha / 2$. The value $t_{1-\alpha / 2 ; n-1}$ is also sometimes called the critical value for the test. The two decision rules for the test are as follows. (1) If $\left|t^{*}\right|>t_{1-\alpha / 2 ; n-1}$, then the null hypothesis is rejected. That is, if $\left|t^{*}\right|>t_{1-\alpha / 2 ; n-1}$, then the signature for chemical $j$ is said to be detected. (2) If $\left|t^{*}\right| \leq t_{1-\alpha / 2 ; n-1}$, then the signature for chemical $j$ is said to be undetected. For the remainder of this report, the term detected is defined as in Defintion 1. This definition may be refined in future work.

Definition 1: (Detected) The signature for chemical $j$ is detected in a plume if its associated 
regression coefficient, $\beta_{j}$, is statistically significant based on a two-tailed level $\alpha$ hypothesis test.

The level of the test, $\alpha$, controls the probability of a false alarm (also called a Type I Error). In this scenario, a false alarm occurs when the signature for chemical $j$ is detected by the test when, in truth, the signature is not present. Obversely, the probability that the test correctly does not detect the chemical signature is $1-\alpha$. Typically, $\alpha$ is selected by the researcher. Common values of $\alpha$ are $0.01,0.05$, or 0.10 , which correspond to $1 \%, 5 \%$, or $10 \%$ false alarm probabilities.

Here, a distinction is made between the terms detection and identification. As detailed in Definition 1, the signature for a chemical is detected if its associated regression coefficient is statistically significant. However, the definition is not designed to imply that the chemical itself has been identified. This distinction is made because two different chemicals with similar absorbance spectra can lead to similar chemical signatures. In cases like these, both of the chemical signatures may have statistically significant regression coefficients. Thus, while the chemical signatures for both chemicals have been detected, neither chemical has been identified as the chemical in the plume.

\subsection{The Power of the Test}

As stated earlier, the probability that the test does not detect the chemical signature when, in truth, the chemical signature is not present is $1-\alpha$. Now, suppose one is interested in finding out the probability that the test detects the chemical signature when, in truth, the chemical signature is present at some level. Intuition dictates that as a chemical's signal strength increases (relative to the amount of background noise), the probability of detecting that chemical's signature increases as well. In fact, one can compute the probability that a hypothesis test correctly detects a given chemical's signature; this is known as the power of the test.

The power of a hypothesis test is the probability that the test rejects the null hypothesis when, in truth, the alternative hypothesis is true [7]. In the context of this report, the power of the test is the probability that the test detects the signature for a chemical when, 
in truth, the signature is present; that is:

$$
\operatorname{Power}\left(k ; \gamma_{j}\right)=\operatorname{Pr}\left(\text { Reject } H_{o}: \beta_{j}=0 \mid H_{a}: \beta_{j}=k \text { is true } ; \gamma_{j}\right)
$$

where the vertical line "|" is read as "given that." Power is viewed as a function of $k$ (the true amount of chemical signature), and $\gamma_{j}$ (the variance term defined in Equation (17), which is a function of $\boldsymbol{\tau}_{a}, T_{p}, T_{g}, \boldsymbol{\epsilon}_{g}, \mathbf{A}_{j}$, and $\Sigma_{g}$ ).

It is important to note that the power function can be computed prior to data collection. As shown earlier, the test power is a function of $\boldsymbol{\tau}_{a}, T_{p}, T_{g}, \boldsymbol{\epsilon}_{g}, \mathbf{A}_{j}$, and $\Sigma_{g}$. Parameters $\boldsymbol{\tau}_{a}, T_{p}, T_{g}$ and $\boldsymbol{\epsilon}_{g}$ can be estimated or selected based on the nature of the mission plan. Additionally, if some prior estimate for $\Sigma_{g}$ is available (i.e. from a covariance library that holds data collected from previous fly-bys at the mission sites of interest, or data from a similar site), then, the power function can be computed for various choices of chemicals and background materials. Even if an informative estimate for $\Sigma_{g}$ is not available, an identity matrix can be used as a temporary estimate. Of particular interest is the fact that with $k$ held constant, the power function can be computed for various choices of background, and the background associated with the highest power can be thought of as the best background for detection since the relative probability that the chemical will be detected on that background is the highest. With this in mind, the power function is interrogated further.

Since the null hypothesis is rejected if $\left|t^{*}\right|>t_{1-\frac{\alpha}{2} ; n-1}$, the power function in Equation (21) can be expressed as follows:

$$
\begin{aligned}
& \text { Power }\left(k ; \gamma_{j}\right) \\
& =\operatorname{Pr}\left(\left|t^{*}\right|>t_{1-\frac{\alpha}{2}, n-1} \mid H_{a}: \beta_{j}=k \text { is true } ; \gamma_{j}\right) \\
& =\operatorname{Pr}\left(t^{*}>t_{1-\frac{\alpha}{2}, n-1} \mid H_{a}: \beta_{j}=k \text { is true; } \gamma_{j}\right)+\operatorname{Pr}\left(t^{*}<-t_{1-\frac{\alpha}{2}, n-1} \mid H_{a}: \beta_{j}=k \text { is true; } \gamma_{j}\right) \\
& =1-\operatorname{Pr}\left(t^{*} \leq t_{1-\frac{\alpha}{2}, n-1} \mid H_{a}: \beta_{j}=k \text { is true } \gamma_{j}+\operatorname{Pr}\left(t^{*}<-t_{1-\frac{\alpha}{2}, n-1} \mid H_{a}: \beta_{j}=k \text { is true; } \gamma_{j}\right) .\right.
\end{aligned}
$$

The distribution of $t^{*}$ depends on which hypothesis $\left(H_{o}\right.$ or $\left.H_{a}\right)$ is true. When the null hypothesis is true (i.e. when $H_{o}: \beta_{j}=0$ is true), $t^{*}$ follows a $t$-distribution with $d f=n-1$. When the alternative hypothesis is true (generically written as $H_{a}: \beta_{j}=k$ for some non-zero value $k), t^{*}$ follows a noncentral $t$-distribution with $d f=n-1$ and noncentrality parameter $|k| / \sqrt{\gamma_{j}}$ where where $\gamma_{j}=\left(\mathbf{x}_{j}^{\prime} \Sigma_{g}^{-1} \mathbf{x}_{j}\right)^{-1}$ is as defined in Equation (17) [7]. 
Let $\Psi(\cdot ; d f, \delta)$ be the cumulative distribution function (cdf) for the noncentral $t$-distribution with $d f$ degrees of freedom and noncentrality parameter $\delta$. Here, the symbol $\Psi$ is used instead of a capital $T$ in order to distinguish the cdf from the notation for temperature. Then, the power function in Equation (22) can be expressed as

$$
\operatorname{Power}\left(k ; \gamma_{j}\right)=1-\Psi\left(t_{1-\alpha / 2 ; n-1} ; n-1,|k| / \sqrt{\gamma_{j}}\right)+\Psi\left(-t_{1-\alpha / 2 ; n-1} ; n-1,|k| / \sqrt{\gamma_{j}}\right)
$$

Using properties of the noncentral $t$-distribution, it can be shown that:

$$
\begin{aligned}
\lim _{|k| \rightarrow 0} \operatorname{Power}\left(k ; \gamma_{j}\right) & =\lim _{\gamma_{j} \rightarrow \infty} \operatorname{Power}\left(k ; \gamma_{j}\right)=1-\left(1-\frac{\alpha}{2}\right)+\left(\frac{\alpha}{2}\right)=\alpha \\
\lim _{|k| \rightarrow \infty} \operatorname{Power}\left(k ; \gamma_{j}\right) & =\lim _{\gamma_{j} \rightarrow 0} \operatorname{Power}\left(k ; \gamma_{j}\right)=1-0+0=1 .
\end{aligned}
$$

The results of Equations (24) and (25) are entirely expected and reveal the effects that the signal (summarized as $k$ ) and the variance term $\left(\gamma_{j}\right)$ have on the test power, i.e., on the test's ability to detect the signature of a given chemical when the signature is present. Equation (24) shows that when the signal strength decreases relative to the variance term, the test power approaches its minimum value, $\alpha$. In fact, when $k=0$, the test power is equal to $\alpha$ which means that when no chemical signature is present, the test detects the chemical signature with probability $\alpha$. This is consistent with the definition of $\alpha$ since $\alpha$ is the probability of a false positive.

Of particular interest is the relationship between the variance term, $\gamma_{j}$ and the power function. Equation (25) shows that as the variance term decreases relative to signal strength, the probability that the test detects a given chemical's signature increases and approaches 1 . The expression for $\gamma_{j}$ appears in Equation (17); however, it is restated here using a slightly different notation:

$$
\gamma_{j}=\gamma_{j, g}=\left(\mathbf{x}_{j}^{\prime} \Sigma_{g}^{-1} \mathbf{x}_{j}\right)^{-1}
$$

The variance term is written in this way in order to track the effect that background condition " $g$ " has on the power function. Suppose a background condition has associated parameters $\boldsymbol{\tau}_{a}, \boldsymbol{\epsilon}_{g}, T_{g}$, with a plume temperature term $T_{p}$ leading to a variance term denoted $\gamma_{j, 1}$. Then, suppose another background condition has similarly defined parameters leading to a variance term $\gamma_{j, 2}$. Then, it can be shown that:

$$
\text { If } \gamma_{j, 1}<\gamma_{j, 2} \text {, then Power }\left(k ; \gamma_{j, 1}\right)>\operatorname{Power}\left(k ; \gamma_{j, 2}\right) \text {. }
$$


That is, holding $k$ constant, if the variance term associated with background 1 is smaller than the variance term associated with background 2, then, the probability that the test detects the signature for chemical $j$ is larger on background 1 compared to background 2. Thus, this variance term can be used to order or rank the backgrounds from "best" to "worst" in terms of the probability that the test will detect the signature of the chemical of interest.

This last result can be used to construct the preliminary MPT that was described in the introduction of this report. Prior to data collection, variance terms for selected background condition scenarios can be computed. Using these terms, the background conditions can be ranked and the relative "best" background for chemical detection can be identified.

\subsection{Statistics for Background Ranking}

The variance term identified in the previous section can be used to construct a statistic to predict relative chemical detectability across background conditions of interest. Suppose chemical $j$ with absorbance spectrum $\mathbf{A}_{j}$ is of interest. Let $g=1, \ldots, G$ be a set of $G$ background conditions under consideration. For ease of notation, suppose that all of the background conditions share the same $\boldsymbol{\tau}_{a}$ and $T_{p}$. Using Equation (26), the variance term associated with background condition $g$ can be expressed as follows:

$$
\gamma_{j, g}=\left(\left(\boldsymbol{\tau}_{a} \odot\left(\mathbf{B}\left(T_{p}\right)-\boldsymbol{\epsilon}_{g} \odot \mathbf{B}\left(T_{g}\right)\right) \odot \mathbf{A}_{j}\right)^{\prime} \Sigma_{g}^{-1}\left(\boldsymbol{\tau}_{a} \odot\left(\mathbf{B}\left(T_{p}\right)-\boldsymbol{\epsilon}_{g} \odot \mathbf{B}\left(T_{g}\right)\right) \odot \mathbf{A}_{j}\right)\right)^{-1}
$$

As shown in the relationship in Equation (27), relatively small values of $\gamma_{j, g}$ lead to relatively large values of the power function. Thus, relatively large values of $\gamma_{j, g}^{-1}$ lead to relatively large values of the power function. Hence, the following statistic is proposed for background ranking

$$
M_{1, g}=\left(\boldsymbol{\tau}_{a} \odot\left(\mathbf{B}\left(T_{p}\right)-\boldsymbol{\epsilon}_{g} \odot \mathbf{B}\left(T_{g}\right)\right) \odot \mathbf{A}_{j}\right)^{\prime} \widehat{\Sigma}_{g}^{-1}\left(\boldsymbol{\tau}_{a} \odot\left(\mathbf{B}\left(T_{p}\right)-\boldsymbol{\epsilon}_{g} \odot \mathbf{B}\left(T_{g}\right)\right) \odot \mathbf{A}_{j}\right),
$$

where $\Sigma_{g}$ is replaced with some estimate $\widehat{\Sigma}_{g}$. As stated earlier, this estimate may come from a covariance library. Thus, given a set of background conditions indexed by $g$ where $g=1, \ldots, G, M_{1, g}$ can be computed for each background condition. Then, background conditions indexed by $g=1, \ldots, G$ can be ranked from best to worst by ordering the $M_{1, g}$ 's from largest to smallest. 
When estimates for the background covariance matrices are not available, an identity matrix can be used as a temporary estimate leading to the following alternative statistic:

$$
\begin{aligned}
M_{2, g} & =\left(\boldsymbol{\tau}_{a} \odot\left(\mathbf{B}\left(T_{p}\right)-\boldsymbol{\epsilon}_{g} \odot \mathbf{B}\left(T_{g}\right)\right) \odot \mathbf{A}_{j}\right)^{\prime}\left(\boldsymbol{\tau}_{a} \odot\left(\mathbf{B}\left(T_{p}\right)-\boldsymbol{\epsilon}_{g} \odot \mathbf{B}\left(T_{g}\right)\right) \odot \mathbf{A}_{j}\right) \\
& =\sum_{i=1}^{n}\left(\tau_{a}\left(\nu_{i}\right)\right)^{2}\left(B\left(T_{p} ; \nu_{i}\right)-\epsilon_{g}\left(\nu_{i}\right) B\left(T_{g} ; \nu_{i}\right)\right)^{2}\left(A_{j}\left(\nu_{i}\right)\right)^{2}
\end{aligned}
$$

Then, background conditions indexed by $g=1, \ldots, G$ can be ranked from best to worst by ordering the $M_{2, g}$ 's from largest to smallest. That is, a relative large value of $M_{2, g}$ indicates that the chemical of interest has a relative large probability of detection over background condition $g$.

The form of $M_{2}$ is particularly interesting because its structure is in agreement with intuition (and, thus, so is the structure of $M_{1}$ ). The reasoning is as follows. Intuition dictates that it is easier to detect a chemical when (i) the atmospheric interference is small, i.e., when the $\tau_{a}\left(\nu_{i}\right)$ 's are close to 1 , (ii) the $\left(B\left(T_{p} ; \nu_{i}\right)-\epsilon_{g}\left(\nu_{i}\right) B\left(T_{g} ; \nu_{i}\right)\right)$ 's are large in magnitude (as noted in previous work [10]), and (iii) the chemical of interest has a large absorbance spectrum. In fact, (i)-(iii) correspond to the case where $M_{2}$ is large. Thus, justification for the use of statistics $M_{1}$ and $M_{2}$ follows from both a theoretical argument as well as from intuition.

In general, $M_{1}$ and $M_{2}$ will not be of the same scale. However, the concept of background ranking still holds for both statistics. Therefore, prior to embarking on a mission, $M_{1, g}$ or $M_{2, g}$ can be computed in order to predict the background condition over which the $t$-test will have the (relative) highest probability of detecting the signature of the chemical of interest.

\section{Experimental Methods}

\subsection{Overview}

Six scenarios for chemical detection were constructed. Using these scenarios as guides, the InfraRed Systems Analysis in General Environments (IR-SAGE) code [9] was used to construct six simplified LWIR hyperspectral images of gas plumes over organized background pixels. IR-SAGE uses the physics-based model in Equation (6) to simulate a radiance vector from the LWIR band using a chemical absorbance spectrum, a background emissivity 
spectrum, and a given set of atmospheric conditions, while perturbing these quantities with Gaussian noise. For each image, the empirical relative best background for detection is identified. Then, this result is compared to the predictions generated by statistics $M_{1}$ and $M_{2}$.

\subsection{Generation of Synthetic Hyperspectral Images}

Two gases, carbon tetrachloride $\left(\mathrm{CCl}_{4}\right)$ and ammonia $\left(\mathrm{NH}_{3}\right)$ were used for image simulation. Their spectra are presented in Figures 1(a) and 1(b). These spectra are plotted on the same scale in order to highlight the fact that the absorbance spectrum for $\mathrm{CCl}_{4}$ is much larger than that of $\mathrm{NH}_{3}$. The spectrum for $\mathrm{CCl}_{4}$ exhibits one strong peak at around $790 \mathrm{~cm}^{-1}$ while the spectrum for $\mathrm{NH}_{3}$ exhihibits several relatively smaller peaks spread out over the LWIR band. One chemical was inserted into each synthetic image.

A total of six background materials were considered. The corresponding background emissivity spectra are presented in Figure 1(c) and are considered to be representative of asphaltconcrete-soil (ACS), Brick, miscellaneous (MSC), Paint, Snow, and steel-copper-tubing $(S T C O P)$. These spectra are averages of clusters of laboratory-measured individual background materials from the Nonconventional Exploitation Factors Data System (NEFDS), a government database of surface reflection parameters.

In all images, the atmospheric transmissivity was the US 1976 Standard Atmosphere. A plot of the atmospheric transmissivity spectrum appears in Figure 1(d).

Each simulated image has dimension $150 \times 120 \times 126$ (rows by columns by spectral dimension). The spectral dimension is 126 because the LWIR wavenumber range under consideration is defined to be $750 \mathrm{~cm}^{-1}$ to $1250 \mathrm{~cm}^{-1}$ in steps of four. The six background spectra are inserted across the rows in six 25-pixel long swaths. The chemical of interest is inserted as six 20-column wide bands at concentration path-lengths of 16, 8, 4, 2, 1, and 0 ppm-m. This configuration produces 500 pixel replicates within each of the 36 background material/concentration path-length combination. A sample broadband image (i.e. a hyperspectral image averaged over the spectral dimension) appears in Figure 2.

Using IR-SAGE, images were created for three temperature cases: $T_{p}>T_{g}, T_{p}=T_{g}$, and $T_{p}<T_{g}$. In each case, $T_{g}$ was fixed at $300 K$ while $T_{p}$ was allowed to be either $310 K, 300 K$, 
or $290 K$. Simulated zero-mean instrument noise was used to perturb the spectra in each pixel.

\subsection{Analyses Performed on Synthetic Images}

In a basic mission planning scenario (such as the one presented in the introduction of this report), there is one chemical of interest and several background conditions on over which the chemical signature may be detected. Therefore, at a minimum, it is assumed that the chemical absorbance and background emissivity spectra are known and available. Additionally, at this preliminary stage, it is assumed that $\tau_{a}, T_{p}$, and $T_{g}$ are either known or specified by the mission plan.

The GLSE, $\widehat{\beta}_{j}$, from Equation (18) is used as a gas detector where

$$
\widehat{\beta}_{j}=\left(\mathbf{x}_{j}^{\prime} \widehat{\Sigma}_{g}^{-1} \mathbf{x}_{j}\right)^{-1} \mathbf{x}_{j}^{\prime} \widehat{\Sigma}_{g}^{-1} \mathbf{r}
$$

and $\mathbf{x}_{j}=\boldsymbol{\tau}_{a} \odot\left(\mathbf{B}\left(T_{p}\right)-\boldsymbol{\epsilon}_{g} \odot \mathbf{B}\left(T_{g}\right)\right) \odot \mathbf{A}_{j}$. This GLSE is applied to each of the 500 replicates within each background material/concentration path-length combination. For each of the 500 trials, if the GLSE is deemed statistically significant based on a level $\alpha=0.05$ test, then the signature for the chemical of interest is said to be detected in that pixel. For each background material/concentration path-length combination, the number of detections is recorded across the trials and the sample detection proportion, $\widehat{p}$, is computed where

$$
\widehat{p}=\frac{\# \text { of detections }}{500} \text {. }
$$

The statistic, $\widehat{p}$, is treated as an estimate of the gas detection probability. For each image, 36 such proportions are computed. Then, for each background, the empirical detection proportion is plotted as a function of concentration path-length. Examination of these curves reveals the empirical best background material for detection and the empirical ranking of the background materials. Additionally, the empirical detection curves are compared to the predicted power function curves as defined in Equation (23). 


\section{Results and Discussion}

Calculation of $M_{1}$ and the power function requires estimates of the background covariance matrices in each image. Using the IR-SAGE images, a covariance matrix for each background was computed from the $0 \mathrm{ppm}-m$ pixels. These covariance matrices are referred to as the background covariance matrices because they are computed from off-plume pixels. For illustrative purposes, this collection of matrices was treated as the covariance matrix library and this library was used to compute $M_{1}$ and the power function. Effectively, this can be viewed as a best-case scenario, where the library estimate of the covariance matrix is exactly the covariance matrix observed in the scene.

For the results presented in this report, the actual observed data are used to compute $M_{1}$ and the power function. Therefore, the results stemming from $M_{1}$ and the power function are not theoretical predictions in the strictest sense. However, proceeding in this way allows the study of the performance of $M_{1}$ (a statistic that incorporates an informative covariance matrix) and $M_{2}$ a statistic that does not incorporate an informative covariance matrix).

\subsection{Results for the $\mathrm{CCl}_{4}$ Images}

Beginning with the case where $T_{p}=310 \mathrm{~K}$ and $T_{g}=300 \mathrm{~K}$, the results of the analyses performed on the IR-SAGE images containing $\mathrm{CCl}_{4}$ are presented. The background ranking prediction statistics $M_{1}$ and $M_{2}$ were computed for each of the six background materials under consideration and the results are summarized in Figures 3(a) and 3(b). Using Equation (23), the predicted power function for each background material was computed; a plot of the resulting curves appears in Figure 3(c). A plot of the empirical detection curves for each background material appears in Figure 3(d).

Recall that $M_{1}$ (like $M_{2}$ ) is constructed such that if a background material yields a relatively larger value of the statistic, this means that the chemical of interest has a relatively larger chance of being detected on that background material. Examination of Figures 3(a) and 3(b) shows that the prediction statistics agree that Paint is the relative best background material for detection of $\mathrm{CCl}_{4}$ (under the given atmospheric and temperature conditions and under the various assumptions highlighted in Sections 2 and 3). Additionally, $M_{1}$ implies that $A C S$ is the worst background for detection while $M_{2}$ implies that Snow is the worst 
background material for detection. A complete list of the background ranks implied by the statistics appears in Table 1. Consider Figure 3(c). The background ordering implied by $M_{1}$ and by the power function are one-to-one since $M_{1}$ is the (inverse of the) term that was found to drive the power function. It is important to note that the power functions for the various background materials do not cross; this illustrates the fact that given $k$, background materials can be rank-ordered using the associated variance term (or its inverse, $M_{1}$ ).

Examination of the empirical detection curves presented in Figure 3(d) shows that the curve for Paint sits above all others. Thus, Paint is the background material with the highest empirical power and is the empirical best background for detection of $\mathrm{CCl}_{4}$. This is consistent with the predictions obtained from the prediction statistics. It is important to note that unlike the predicted power functions, the empirical detection curves for the various background materials can cross. As a result, the observed background ranking may be different for different choices of concentration path-length (i.e. $k$ ). For the $\mathrm{CCl}_{4}$ data with $T_{p}=310 \mathrm{~K}$ and $T_{g}=300 \mathrm{~K}$, the concentration path-length at which the most background separation is seen is at $k=2 \mathrm{ppm}-\mathrm{m}$. A list of the empirical background ranking for this choice of $k$ appears in Table 1. Based on the empirical ranking, the three best backgrounds for detection are Paint, STCOP, and $M S C$, which is consistent with the predictions from $M_{1}$ and $M_{2}$. This is particularly remarkable because $M_{2}$ does not incorporate an informative covariance matrix.

Next, the results of the analyses performed on the IR-SAGE image that contains $\mathrm{CCl}_{4}$ with $T_{p}=T_{g}=300 \mathrm{~K}$ are presented. Plots of statistics $M_{1}$ and $M_{2}$, the predicted power functions, and the empirical detection curves are presented in Figure 4. For this temperature case, the statistics provide identical predictions for the background rankings (see Table 2) and both statistics agree that Paint is the relative best background material for detection of $\mathrm{CCl}_{4}$. The empirical detection curves displayed in Figure 4(d) clearly demonstrate that Paint is the empirical best background for detection of $\mathrm{CCl}_{4}$. The empirical background rankings at $k=8 \mathrm{ppm}-m$ (the point at which the most background separation is observed) are summarized in Table 2. The predicted background rankings and the empirical background rankings agree on the three best background materials (Paint, STCOP, MSC) and the worst background material (Snow). Thus, again, statistic $M_{2}$ appears to perform as well as statistic $M_{1}$ for these data.

Finally, the results of the analyses performed on the IR-SAGE image that contains $\mathrm{CCl}_{4}$ with 
$T_{p}=290 \mathrm{~K}$ and $T_{g}=300 \mathrm{~K}$ are presented. Plots of statistics $M_{1}$ and $M_{2}$, the predicted power functions, and the empirical detection curves are displayed in Figure 5. A shift is observed: for this temperature case, the predicted best background material for $\mathrm{CCl}_{4}$ is Snow. This is in contrast to the $T_{p} \geq T_{g}$ cases where the predicted best background material was found to be Paint. The empirical background ordering shows the same type of background shifting. The plot in Figure 5(d) shows that the empirical best background material for detection (at $4 \mathrm{ppm}-\mathrm{m}$ ) is Snow. This type of background-rank shifting is consistent with observations made in previous work [10].

The empirical background ranks at $k=4 p p m-m$ are summarized in Table 3 . The empirical ranks at this concentration path-length are the same as the ranks predicted by $M_{1}$. While the ranks implied by $M_{2}$ are not identical to the empirical ranks, they agree on four out of six ranks.

\subsection{Results for the $\mathrm{NH}_{3}$ Images}

Beginning with the case where $T_{p}=310 \mathrm{~K}$ and $T_{g}=300 \mathrm{~K}$, the results of the analyses performed on the IR-SAGE images containing $\mathrm{NH}_{3}$ are presented. Prediction statistics $M_{1}$ and $M_{2}$ were computed and are presented in Figures 6(a) and 6(b); a summary of the resulting predicted background ranks appears in Table 1. For this temperature case, statistics $M_{1}$ and $M_{2}$ disagree on which background material is the best for detection. $M_{1}$ implies that $S T C O P$ is the best background material with $M S C$ following closely in second place. $M_{2}$ reverses this order and identifies $M S C$ as the best background with STCOP following closely in second place.

Examination of the empirical detection curves in Figure 6(d) shows that the empirical best background for detection at $8 \mathrm{ppm}-m$ is $S T C O P$ which is consistent with the prediction provided by $M_{1}$. A summary of the empirical background rankings at $k=8 \mathrm{ppm}-m$ appears in Table 1. For these data, statistic $M_{1}$ accurately predicts the best two and the worst two backgrounds for detection. Generally speaking, statistic $M_{1}$ outperforms statistic $M_{2}$ for this case. Thus, this is a situation where having additional information from the covariance matrix aids in the predictive ability of the statistics.

Next, the results of the analyses performed on the IR-SAGE image that contains $\mathrm{NH}_{3}$ with $T_{p}=T_{g}=300 K$ are presented. Plots of statistics $M_{1}$ and $M_{2}$, the predicted power functions, 
and the empirical detection curves are displayed in Figure 7. For this temperature case, the statistics provide identical predictions for the background rankings (see Table 2) and they both predict that that $M S C$ is the best background material for detection of $\mathrm{NH}_{3}$. The empirical best backround for detection is not entirely clear. Examination of the empirical detection proportion curves in Figure $7(\mathrm{~d})$ shows that the curves for the top two materials, $M S C$ and STCOP, overlap. The greatest amount of background seperation occurs at $k=16$ $p p m-m$ and at this concentration path-length, $M S C$ is the best background for the detection of $\mathrm{NH}_{3}$. A summary of the empirical background rankings at $k=16 \mathrm{ppm}-m$ appears in Table 2. At this concentration path-length, the predicted rankings implied by $M_{1}$ and $M_{2}$ agree with the empirical background rankings.

Finally, the results of the analyses performed on the IR-SAGE image that contains $\mathrm{NH}_{3}$ with $T_{p}=290 K$ and $T_{g}=300 K$ are presented. Plots of statistics $M_{1}$ and $M_{2}$, the predicted power functions, and the empirical detection curves are displayed in Figure 8. As seen in the analyses of the $\mathrm{CCl}_{4}$ data, a shift is seen in the background rankings. For this temperature case, the predicted best background material for detection of $\mathrm{NH}_{3}$ is Snow. For the $T_{p} \geq T_{g}$ cases, the predicted worst background for detection is Snow. Figure 8(d) shows that the empirical best background material for detection is Snow since its detection proportion curve sits above all others. The empirical background ranks at $k=16 \mathrm{ppm}-m$ are summarized in Table 3 and are identical to the predictions implied by statistics $M_{1}$ and $M_{2}$.

\section{Conclusions}

Presented in this report is a preliminary exploration of statistics that can be used to rank background conditions based on chemical detectability. Currently, the statistics incorporate information related to atmosphere, temperature and background emissivity; however, they do not yet incorporate other important factors (such as sensor calibration terms or sensor-specific noise terms) that affect chemical detectability. Future work may include investigations of these terms as well as analyses involving more complicated plume compositions, complex backgrounds, and realistic images. 


\section{Acknowledgements}

The authors thank Dr. Kevin Anderson of the Pacific Northwest National Laboratory for his helpful comments.

\section{References}

[1] T. Burr, B.R. Foy, H. Fry, and B. McVey. Characterizing clutter in the context of detecting weak gaseous plumes in hyperspectral imagery. Sensors, 6:1587-1615, 2006.

[2] T. Burr and N Hengartner. Overview of physical models and statistical approaches for weak gaseous plume detection using passive infrared hyperspectral imagery. Sensors, 6:1721-1750, 2006.

[3] N. Gallagher, D. Sheen, J. Shaver, B. Wise, and J. Shultz. Estimation of trace vapor concentration-pathlength in plumes for remote sensing applications from hyperspectral images. Proc. SPIE, 5093:184-194, 2003.

[4] P. Heasler, C. Posse, J. Hylden, and K. Anderson. Nonlinear bayesian algorithms for gas plume detection and estimation from hyper-spectral thermal image data. Sensors, 7:905-920, 2007.

[5] K.N. Liou. An Introduction to Atmospheric Radiation. Academic Press, 2002, $2^{\text {nd }}$ edition.

[6] D Messinger, C. Salvaggio, and N. Sinisgally. Detection of gaseous effluents from airborned lwir hyperspectral imagery using physics-based signatures. International Journal of High Speed Electronics and Systems., 17(6):801-812, 2007.

[7] J. Neter, M. Kutner, C. Nachtsheim, and W. Wasserman. Applied Linear Statistical Models. McGraw-Hill, 1996, $4^{\text {th }}$ edition.

[8] J. Schott. Remote Sensing. Oxford Press:New York, 1997.

[9] D.M. Sheen, N.B. Gallagher, P.G. Heasler, J.F. Schultz, B.M. Wise, S.W. Sharpe, and K.K. Anderson. Infrared chemical detection systems modeling and advanced chemometric analysis. Technical report, 2001. Official Use Only Report. 
[10] S.J. Walsh, M.F. Tardiff, L.K. Chilton, and C.N. Metoyer. Effect of background emissivity on gas detection in thermal hyperspectral imagery. Technical report, 2008. 


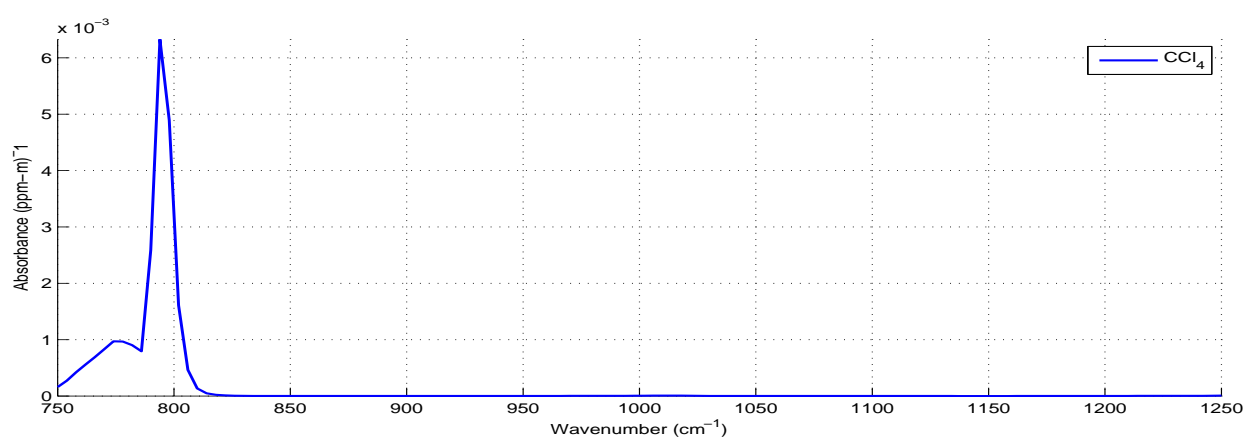

(a)

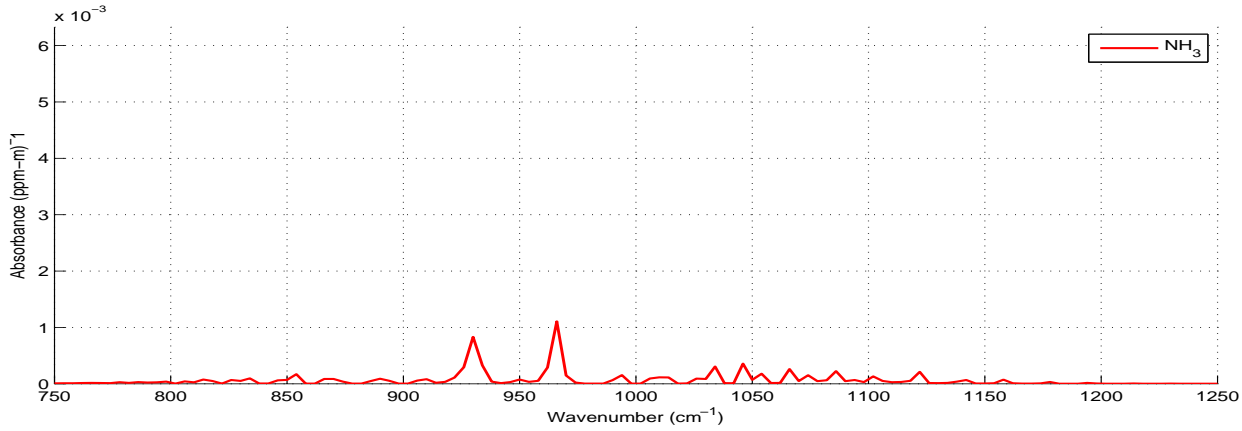

(b)

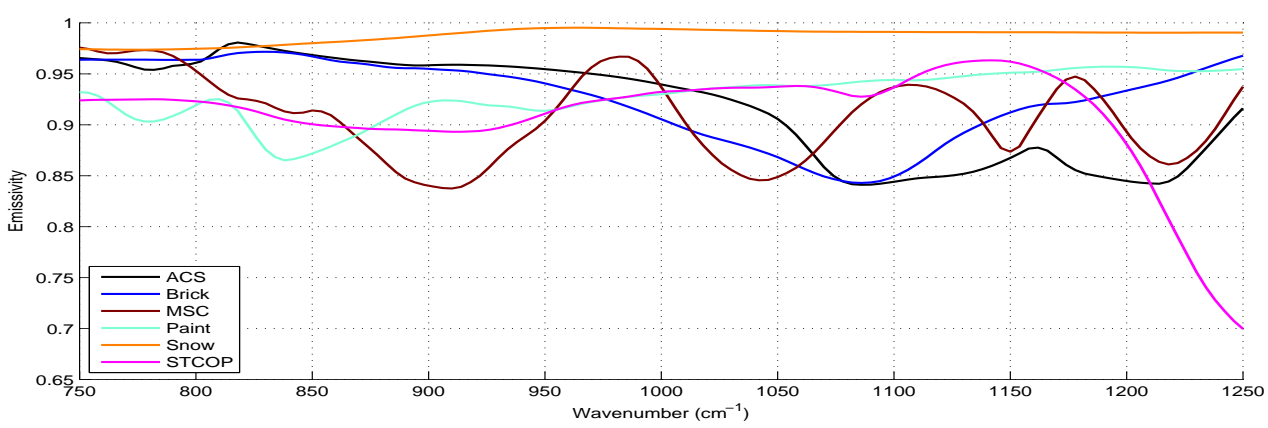

(c)

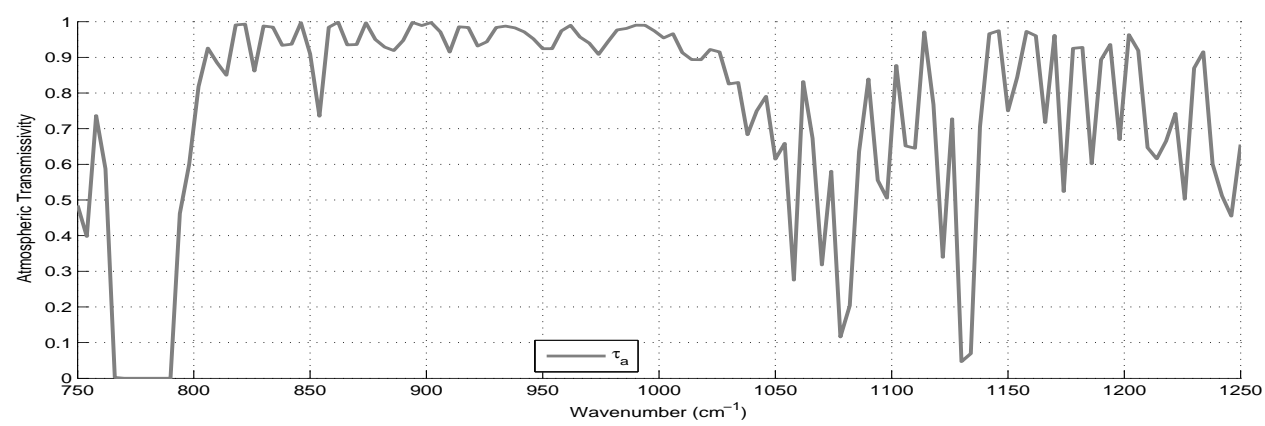

(d)

Figure 1: (a) Absorbance spectrum for $\mathrm{CCl}_{4}$, (b) Absorbance spectrum for $\mathrm{NH}_{3}$, Representative emissivity spectra for six selected materials, and (d) 1976 US Standard atmospheric transmissivity spectrum. 


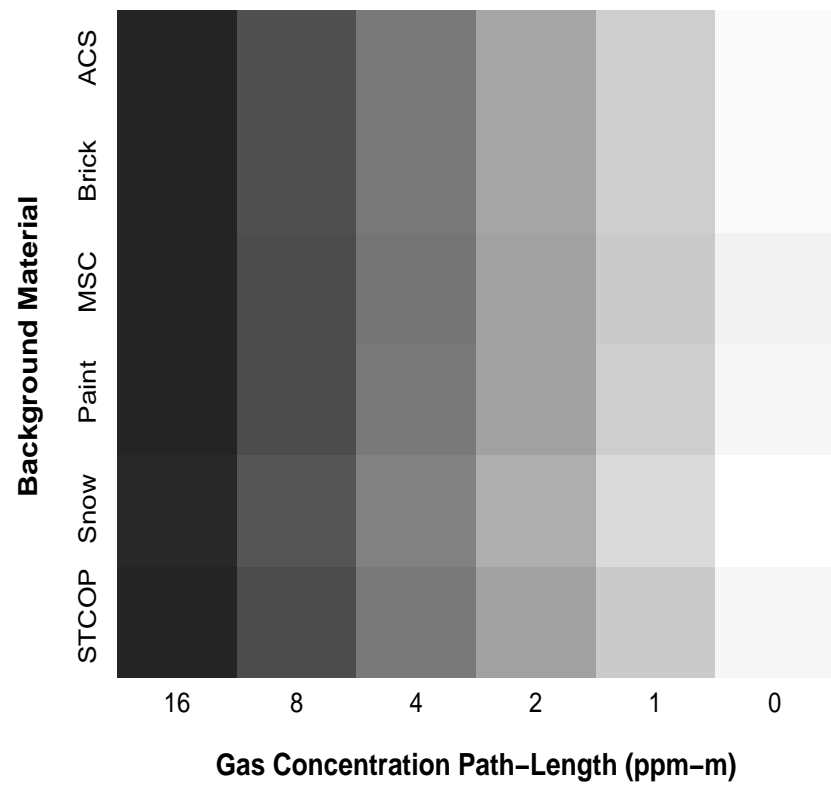

Figure 2: Example of a broadband image for $\mathrm{CCl}_{4}$ using synthetic data from IR-SAGE. This image has been enhanced to better show the gradations between background materials and concentration path-lengths. 


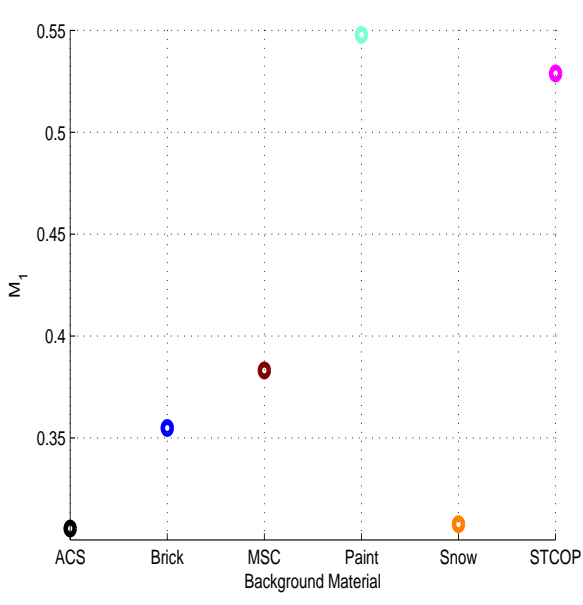

(a)

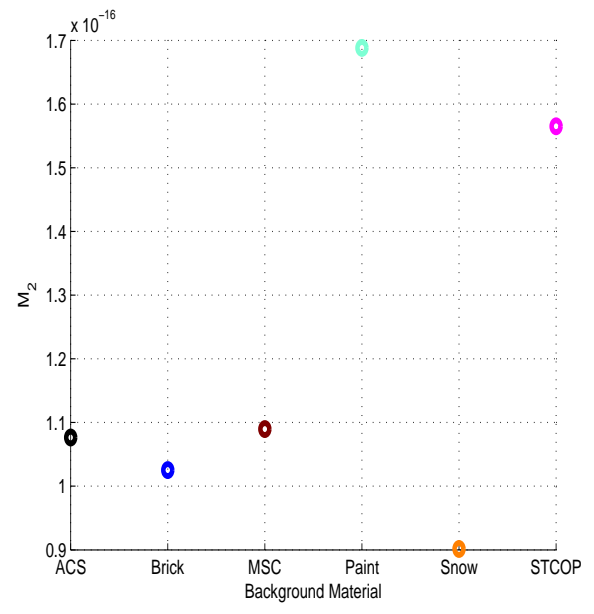

(b)

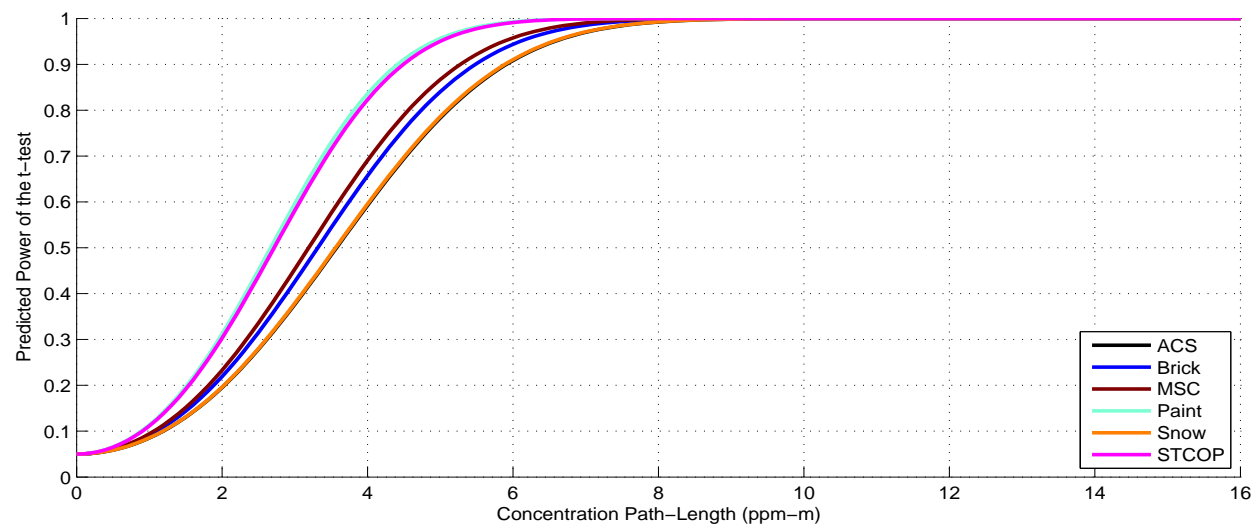

(c)

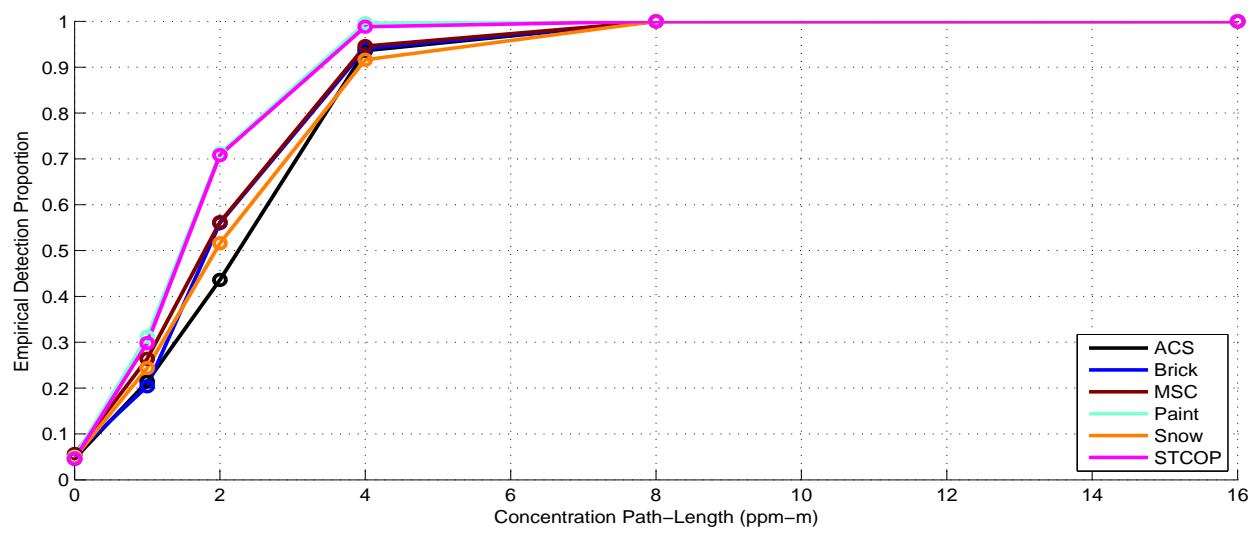

(d)

Figure 3: Graphical results for the IR-SAGE images containing $\mathrm{CCl}_{4}$ with $T_{p}=310 \mathrm{~K}$ and $T_{g}=300 K$ : (a) $M_{1}$ versus background material, (b) $M_{2}$ versus background material, (c) predicted power function, and (d) empirical detection curves. Note that plots (a) and (c) incorporate covariance matrices computed from the $0 \mathrm{ppm}-m$ pixels. 


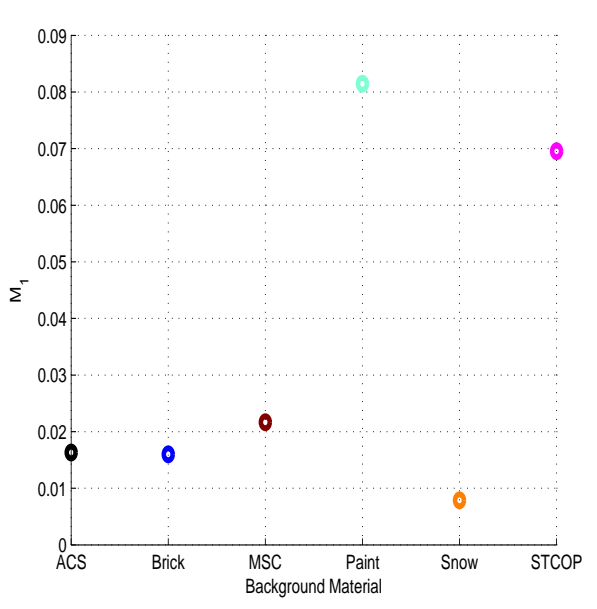

(a)

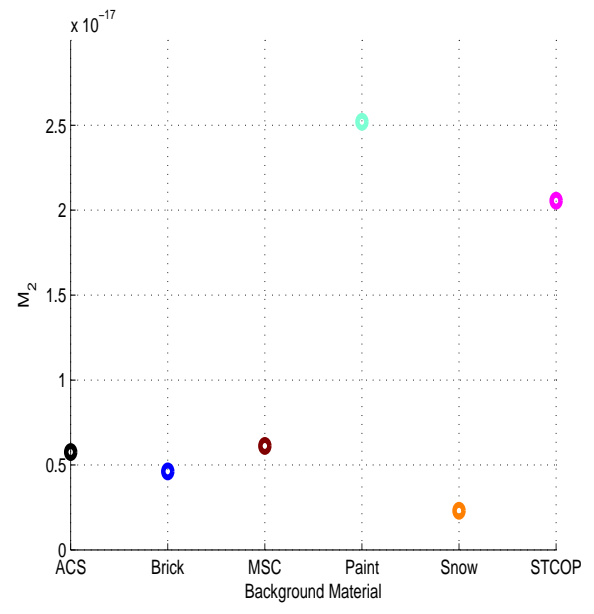

(b)

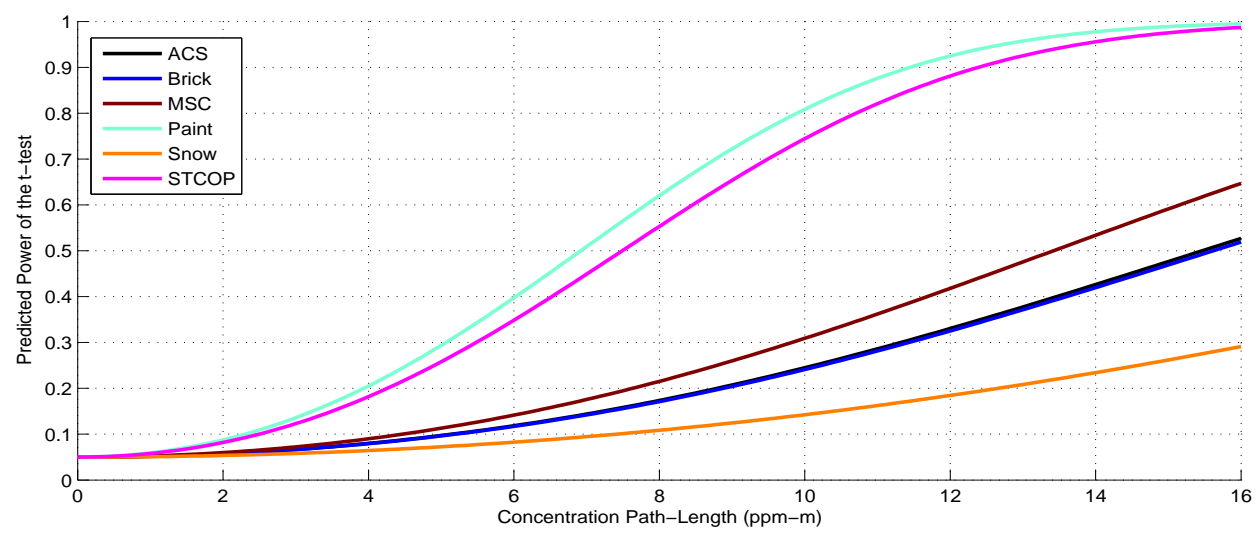

(c)

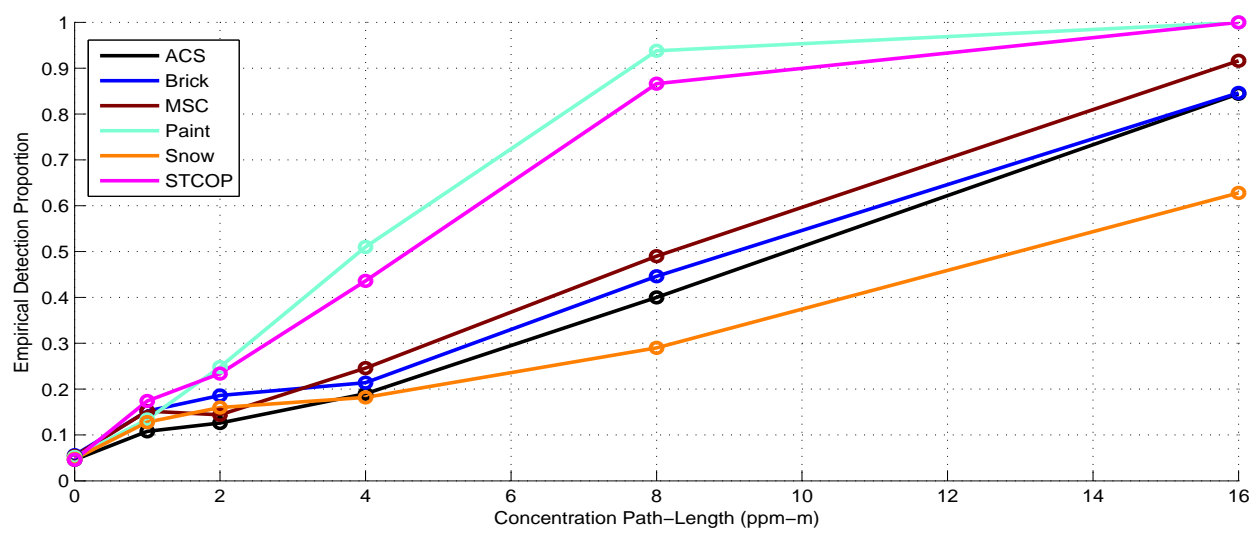

(d)

Figure 4: Graphical results for the IR-SAGE images containing $\mathrm{CCl}_{4}$ with $T_{p}=T_{g}=$ $300 K$ : (a) $M_{1}$ versus background material, (b) $M_{2}$ versus background material, (c) predicted power function, and (d) empirical detection curves. Note that plots (a) and (c) incorporate covariance matrices computed from the $0 \mathrm{ppm}-m$ pixels. 


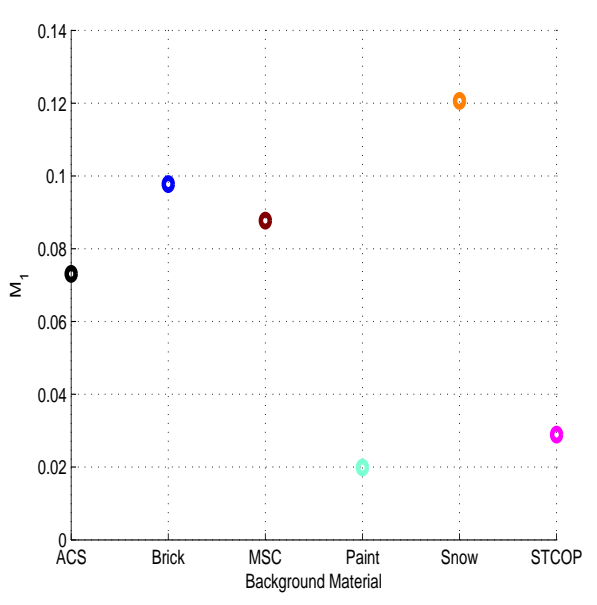

(a)

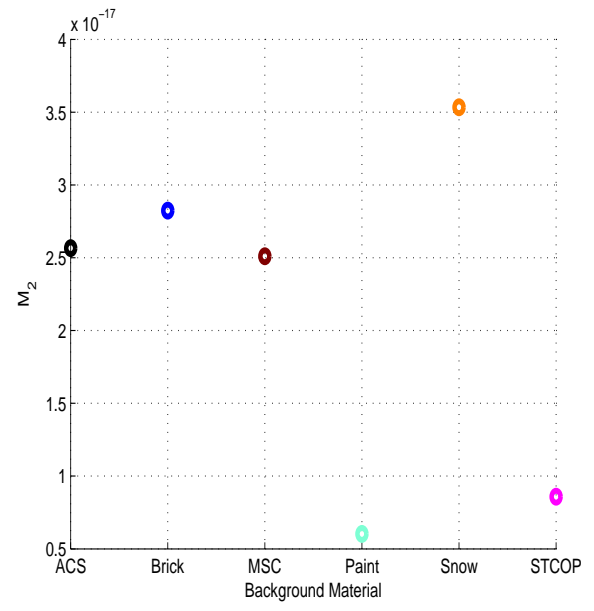

(b)

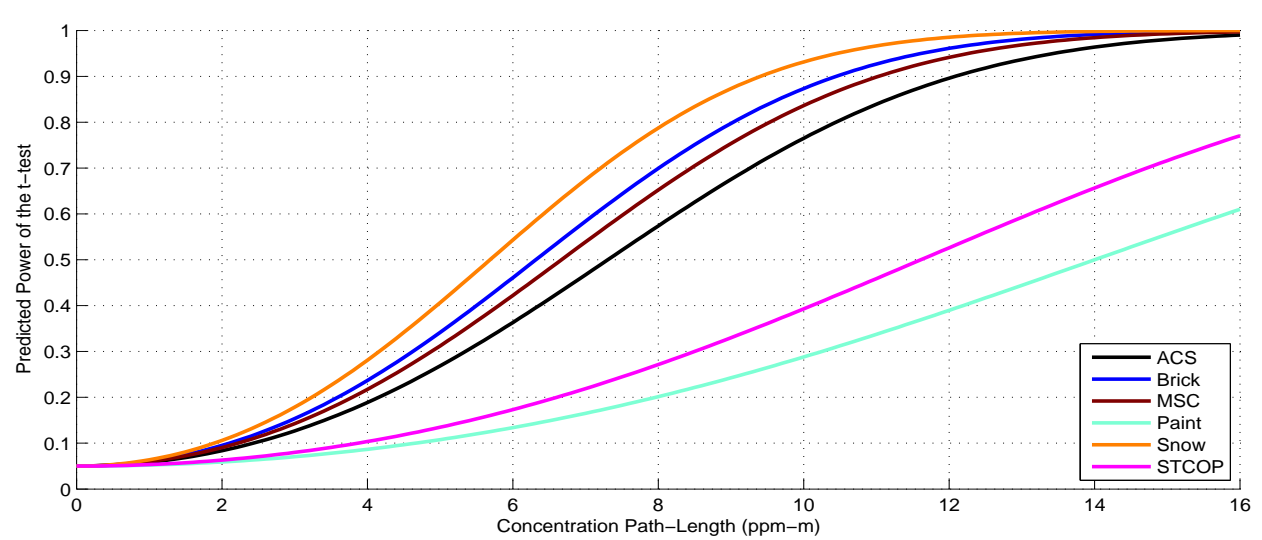

(c)

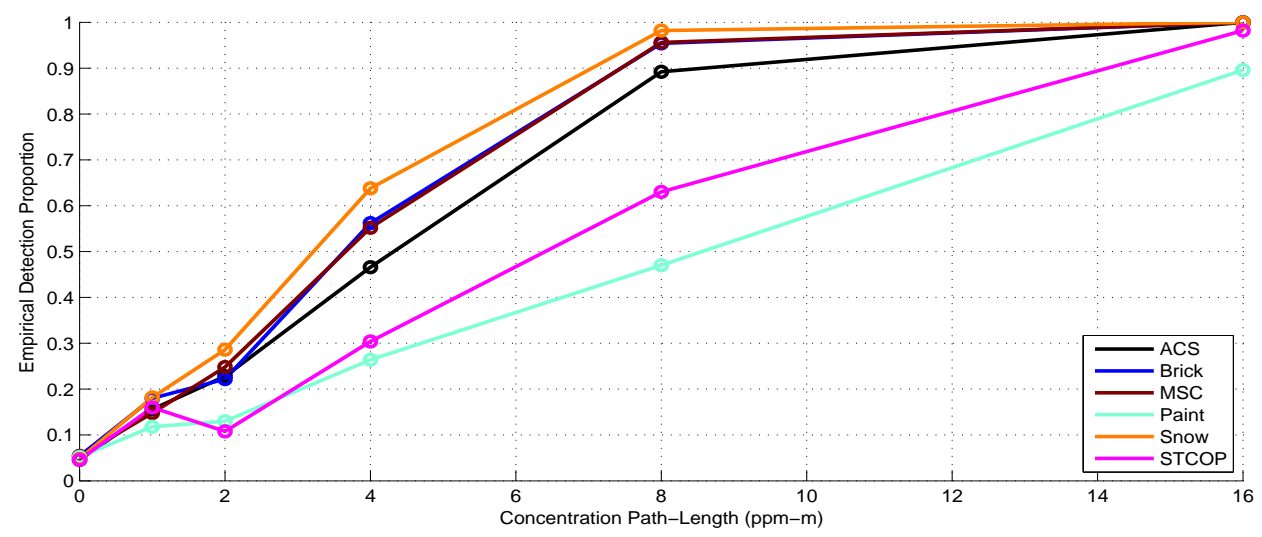

(d)

Figure 5: Graphical results for the IR-SAGE images containing $\mathrm{CCl}_{4}$ with $T_{p}=290 \mathrm{~K}$ and $T_{g}=300 \mathrm{~K}$ : (a) $M_{1}$ versus background material, (b) $M_{2}$ versus background material, (c) predicted power function, and (d) empirical detection curves. Note that plots (a) and (c) incorporate covariance matrices computed from the $0 \mathrm{ppm}-m$ pixels. 


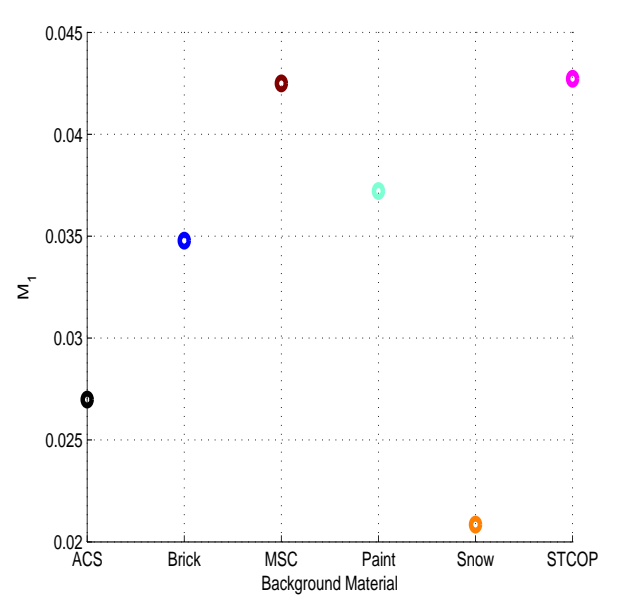

(a)

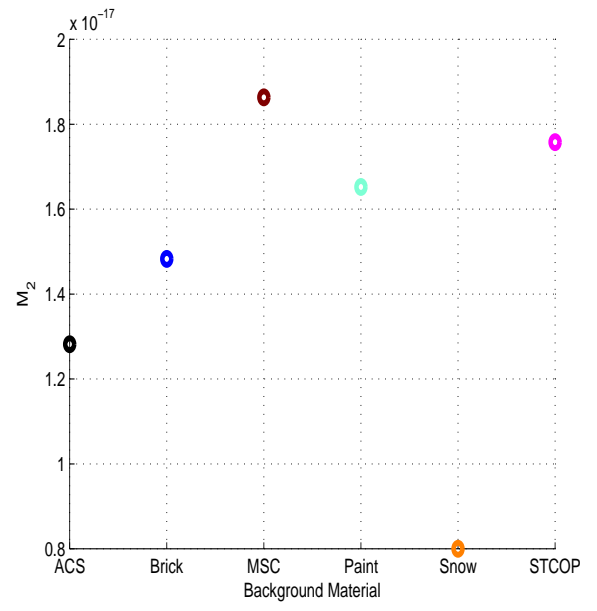

(b)

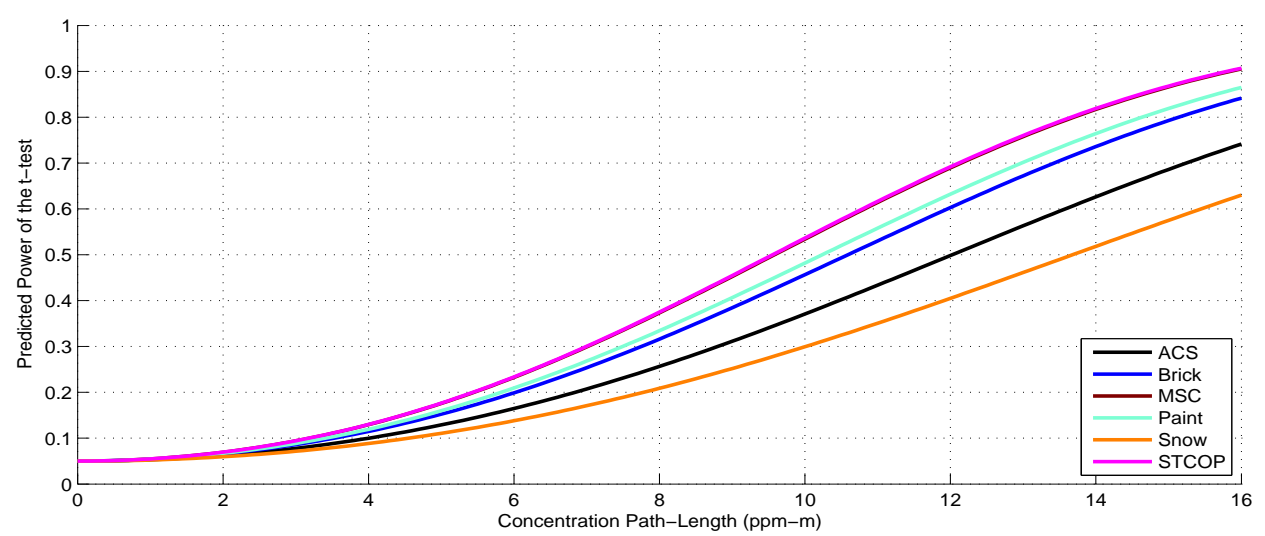

(c)

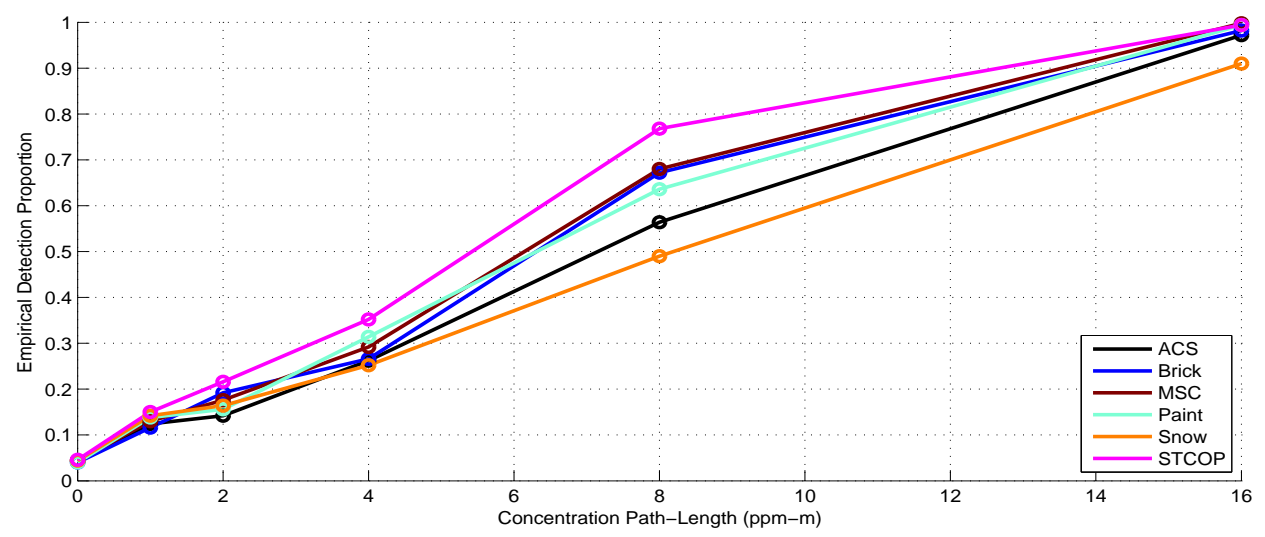

(d)

Figure 6: Graphical results for the IR-SAGE images containing $\mathrm{NH}_{3}$ with $T_{p}=310 \mathrm{~K}$ and $T_{g}=300 \mathrm{~K}$ : (a) $M_{1}$ versus background material, (b) $M_{2}$ versus background material, (c) predicted power function, and (d) empirical detection curves. Note that plots (a) and (c) incorporate covariance matrices computed from the $0 \mathrm{ppm}-m$ pixels. 


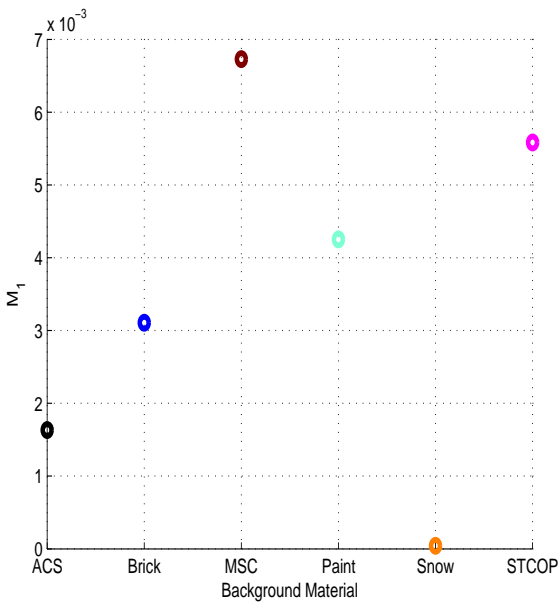

(a)

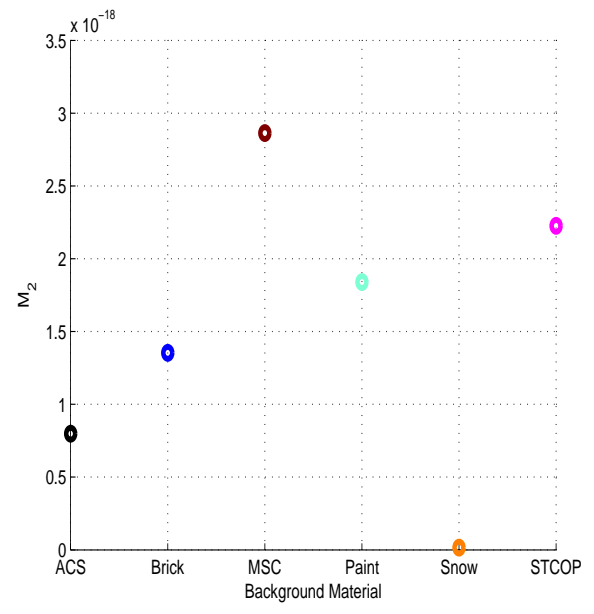

(b)

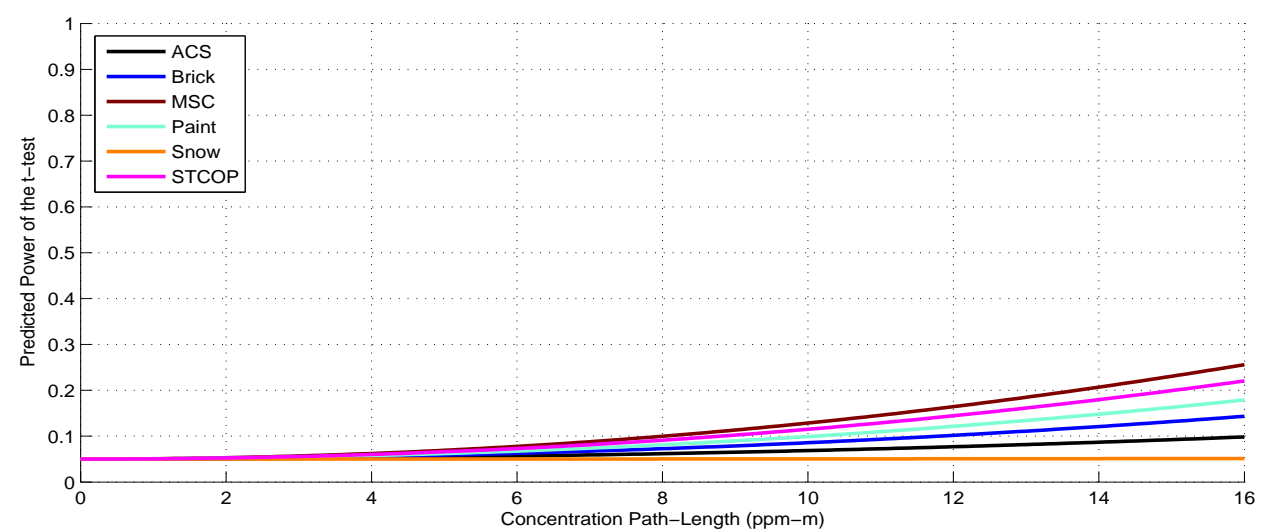

(c)

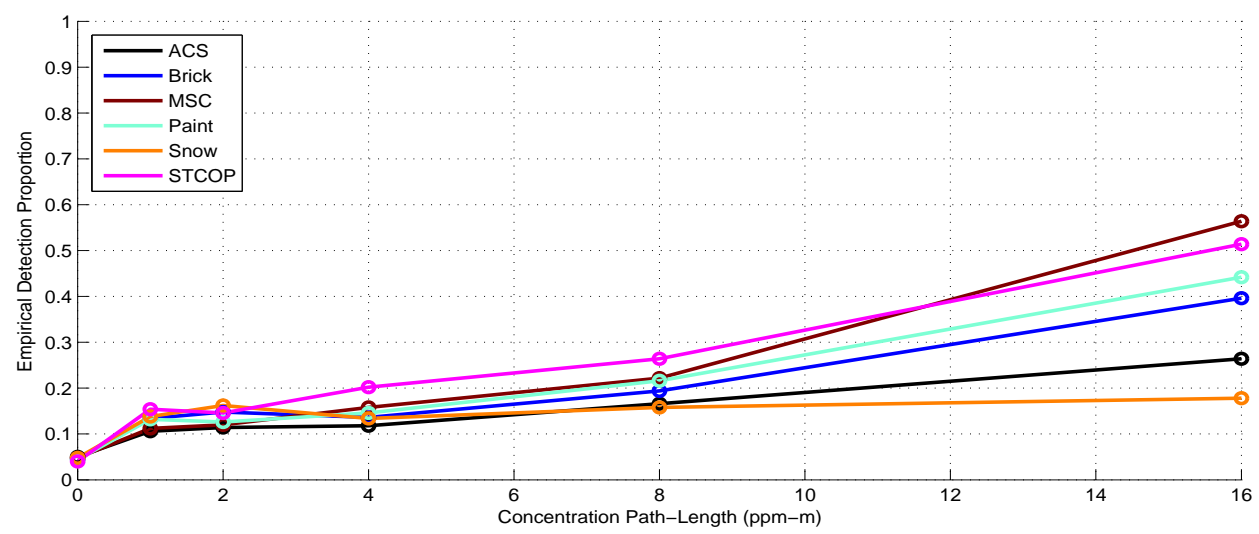

(d)

Figure 7: Graphical results for the IR-SAGE images containing $\mathrm{NH}_{3}$ with $T_{p}=T_{g}=300 \mathrm{~K}$ : (a) $M_{1}$ versus background material, (b) $M_{2}$ versus background material, (c) predicted power function, and (d) empirical detection curves. Note that plots (a) and (c) incorporate covariance matrices computed from the $0 \mathrm{ppm}-m$ pixels. 


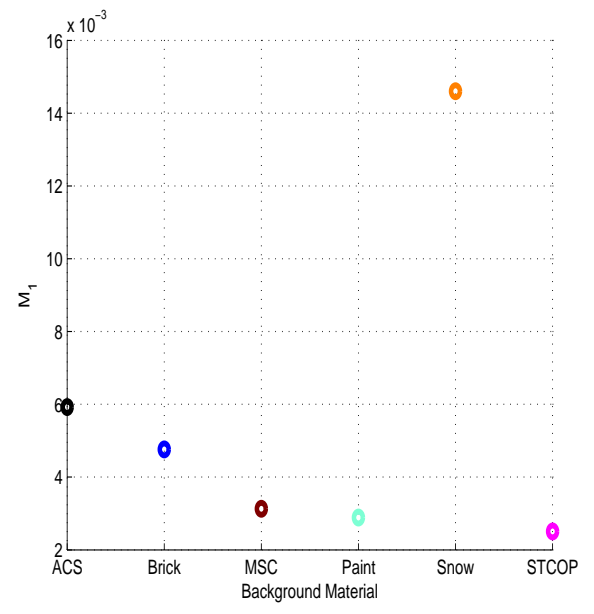

(a)

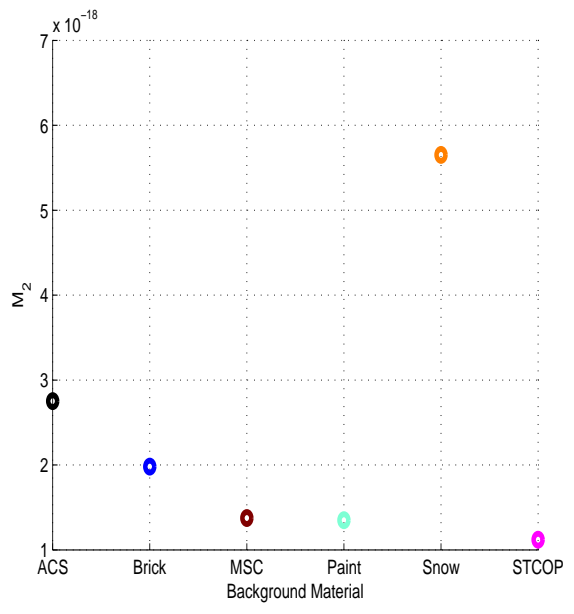

(b)

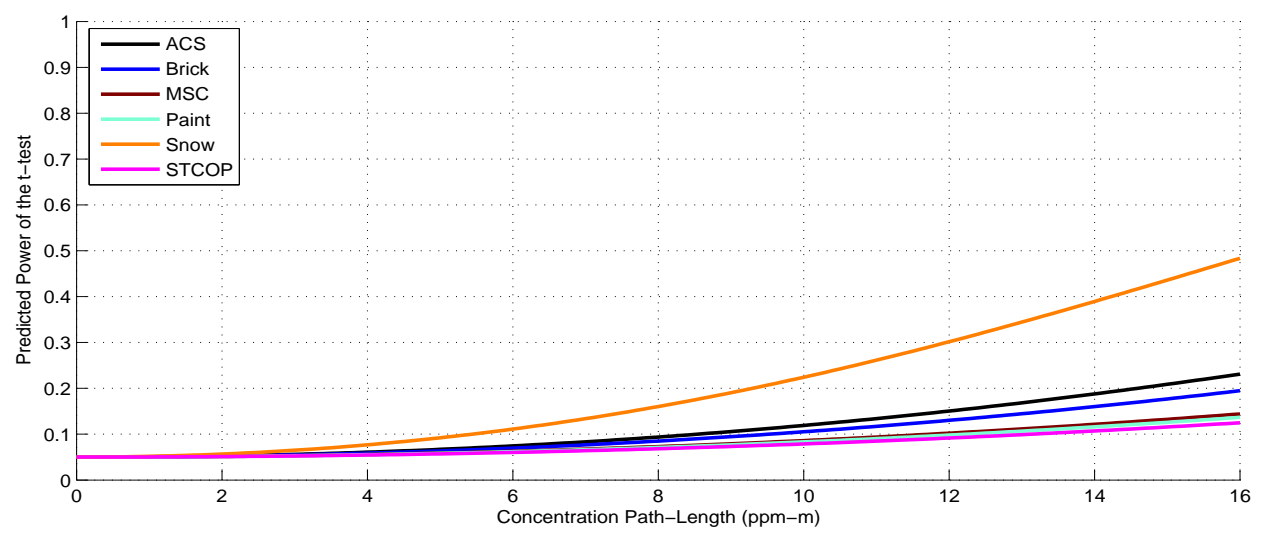

(c)

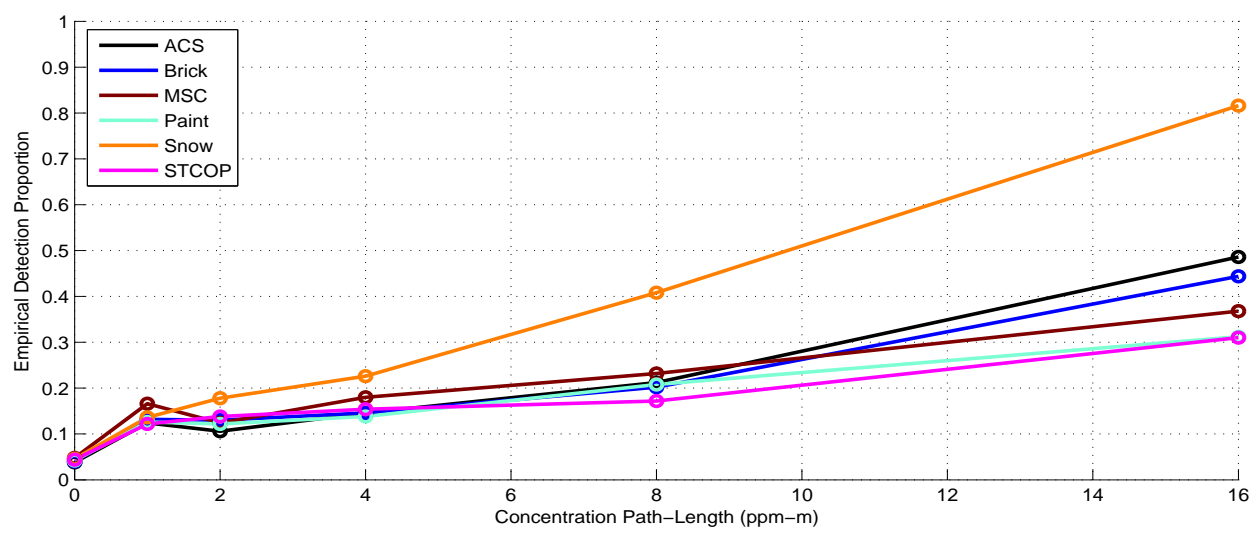

(d)

Figure 8: Graphical results for the IR-SAGE images containing $\mathrm{NH}_{3}$ with $T_{p}=290 \mathrm{~K}$ and $T_{g}=300 \mathrm{~K}$ : (a) $M_{1}$ versus background material, (b) $M_{2}$ versus background material, (c) predicted power function, and (d) empirical detection curves. Note that plots (a) and (c) incorporate covariance matrices computed from the $0 \mathrm{ppm}-m$ pixels. 


\begin{tabular}{|c|c|c|c||c|c|c|}
\hline & \multicolumn{3}{|c||}{$\mathrm{CCl}_{4}$} & \multicolumn{3}{c|}{$\mathrm{NH}_{3}$} \\
\hline Rank & $M_{1}$ & $M_{2}$ & Emp. $(k=2 \mathrm{ppm}-\mathrm{m})$ & $M_{1}$ & $M_{2}$ & Emp. $(k=8 \mathrm{ppm}-\mathrm{m})$ \\
\hline 1 & Paint & Paint & Paint & STCOP & MSC & STCOP \\
\hline 2 & STCOP & STCOP & STCOP & MSC & STCOP & MSC \\
\hline 3 & MSC & MSC & MSC & Paint & Paint & Brick \\
\hline 4 & Brick & ACS & Brick & Brick & Brick & Paint \\
\hline 5 & Snow & Brick & Snow & ACS & ACS & ACS \\
\hline 6 & ACS & Snow & ACS & Snow & Snow & Snow \\
\hline
\end{tabular}

Table 1: Background ranking ( $1=$ best, $6=$ worst) implied by prediction metric $M_{1}$, prediction metric $M_{2}$, and the empirical detection proportions (for selected values of $k$ ) for $\mathrm{CCl}_{4}$ and $\mathrm{NH}_{3}$ where $T_{p}=310 K$ and $T_{g}=300 K$.

\begin{tabular}{|c|c|c|c||c|c|c|}
\hline & \multicolumn{3}{|c||}{$\mathrm{CCl}_{4}$} & \multicolumn{2}{c|}{$\mathrm{NH}_{3}$} \\
\hline Rank & $M_{1}$ & $M_{2}$ & Emp. $(k=8 \mathrm{ppm}-\mathrm{m})$ & $M_{1}$ & $M_{2}$ & Emp. $(k=16 \mathrm{ppm}-\mathrm{m})$ \\
\hline 1 & Paint & Paint & Paint & MSC & MSC & MSC \\
\hline 2 & STCOP & STCOP & STCOP & STCOP & STCOP & STCOP \\
\hline 3 & MSC & MSC & MSC & Paint & Paint & Paint \\
\hline 4 & ACS & ACS & Brick & Brick & Brick & Brick \\
\hline 5 & Brick & Brick & ACS & ACS & ACS & ACS \\
\hline 6 & Snow & Snow & Snow & Snow & Snow & Snow \\
\hline
\end{tabular}

Table 2: Background ranking ( $1=$ best, $6=$ worst) implied by prediction metric $M_{1}$, prediction metric $M_{2}$, and the empirical detection proportions (for selected values of $k$ ) for $\mathrm{CCl}_{4}$ and $\mathrm{NH}_{3}$ where $T_{p}=T_{g}=300 \mathrm{~K}$.

\begin{tabular}{|c|c|c|c||c|c|c|}
\hline & \multicolumn{3}{|c||}{$\mathrm{CCl}_{4}$} & \multicolumn{2}{c|}{$\mathrm{NH}_{3}$} \\
\hline Rank & $M_{1}$ & $M_{2}$ & Emp. $(k=4 \mathrm{ppm}-\mathrm{m})$ & $M_{1}$ & $M_{2}$ & Emp. $(k=16 \mathrm{ppm}-\mathrm{m})$ \\
\hline 1 & Snow & Snow & Snow & Snow & Snow & Snow \\
\hline 2 & Brick & Brick & Brick & ACS & ACS & ACS \\
\hline 3 & MSC & ACS & MSC & Brick & Brick & Brick \\
\hline 4 & ACS & MSC & ACS & MSC & MSC & MSC \\
\hline 5 & STCOP & STCOP & STCOP & Paint & Paint & Paint \\
\hline 6 & Paint & Paint & Paint & STCOP & STCOP & STCOP \\
\hline
\end{tabular}

Table 3: Background ranking ( 1 =best, $6=$ worst) implied by prediction statistic $M_{1}$, prediction statistic $M_{2}$, and the empirical detection proportions (for selected values of $k$ ) for $\mathrm{CCl}_{4}$ and $\mathrm{NH}_{3}$ where $T_{p}=290 \mathrm{~K}$ and $T_{g}=300 \mathrm{~K}$. 